\title{
¿Shallow Cumulus Properties as Captured by Adiabatic Fraction in High-Resolution LES Simulations $\mathscr{0}$
}

\author{
Eshkol Eytan, ${ }^{\mathrm{a}}$ Alexander Khain, ${ }^{\mathrm{b}}$ MARk Pinsky, ${ }^{\mathrm{b}}$ Orit Altaratz, ${ }^{\mathrm{a}} \mathrm{JACOB}$ ShPund, ${ }^{\mathrm{c}}$ And IlAn Koren $^{\mathrm{a}}$ \\ ${ }^{\mathrm{a}}$ Department of Earth and Planetary Science, Weizmann Institute of Science, Rehovot, Israel \\ ${ }^{\mathrm{b}}$ Institute of Earth Science, Hebrew University, Jerusalem, Israel \\ ${ }^{\mathrm{c}}$ Pacific Northwest National Laboratory, Richland, Washington
}

(Manuscript received 24 July 2021, in final form 30 October 2021)

\begin{abstract}
Shallow convective clouds are important players in Earth's energy budget and hydrological cycle, and are abundant in the tropical and subtropical belts. They greatly contribute to the uncertainty in climate predictions due to their unresolved, complex processes that include coupling between the dynamics and microphysics. Analysis of cloud structure can be simplified by considering cloud motions as a combination of moist adiabatic motions like adiabatic updrafts and turbulent motions leading to deviation from adiabaticity. In this work, we study the sizes and occurrence of adiabatic regions in shallow cumulus clouds during their growth and mature stages, and use the adiabatic fraction (AF) as a continuous metric to describe cloud processes and properties from the core to the edge. To do so, we simulate isolated trade wind cumulus clouds of different sizes using the System of Atmospheric Modeling (SAM) model in high resolution $(10 \mathrm{~m})$ with the Hebrew University spectral bin microphysics (SBM). The fine features in the clouds' dynamics and microphysics, including small near-adiabatic volumes and a thin transition zone at the edge of the cloud $(\sim 20-40 \mathrm{~m}$ in width), are captured. The $\mathrm{AF}$ is shown to be an efficient measure for analyzing cloud properties and key processes determining the droplet-size distribution formation and shape during the cloud evolution. Physical processes governing the properties of droplet size distributions at different cloud regions (e.g., core, edge) are analyzed in relation to AF.
\end{abstract}

SIGNIFICANCE STATEMENT: 1) This study investigates the evolution of cumulus clouds (Cu) using a 10-m-resolution LES model with spectral bin microphysics. 2) The study improves the understanding of the mutual effects of adiabatic updrafts and lateral entrainment and mixing. 3) The study demonstrates the existence of an adiabatic core in nonprecipitating $\mathrm{Cu}$. 4) Shapes of the droplet size distributions are closely related to the adiabatic fraction values. 5) Utilization of high resolution reveals the existence of physically significant small features in the cloud structure, such as a narrow cloud interface zone and small adiabatic volumes.

KEYWORDS: Mixing; Cloud microphysics; Cumulus clouds; Large eddy simulations

\section{Introduction}

Clouds are dominant players in Earth's energy budget and hydrological cycle. They affect both the incoming and outgoing radiative fluxes, and the distribution of energy via latent and sensible heat fluxes (Trenberth et al. 2009; Bony et al. 2015). Their important role, together with their turbulent and multiscale nature, makes them a major cause of uncertainty in climate models. They may pose an anthropogenic radiative forcing through their sensitivity to aerosols loading, which is most likely to be negative (cooling) (IPCC 2013). On the other hand, they have feedbacks on a changing climate, which recent research has shown to be positive (i.e., increased surface temperatures will force changes on clouds that will warm the atmosphere) (e.g.,

\footnotetext{
Denotes content that is immediately available upon publication as open access.
}

\footnotetext{
O) Supplemental information related to this paper is available a the Journals Online website: https://doi.org/10.1175/JAS-D-210201.s1.

Corresponding author: Ilan Koren, ilan.koren@weizmann.ac.il
}

Ceppi et al. 2017; Nuijens and Siebesma 2019; Zelinka et al. 2020). Small cumulus clouds $(\mathrm{Cu})$ on the scale of hundreds of meters pose a great challenge, as they constitute a large fraction of the clouds' area and number in the field (i.e., cloud size distribution is a power law; Cahalan and Joseph 1989; Neggers et al. 2003; Rodts et al. 2003; Koren et al. 2008), but are mostly overlooked by the coarse resolution of current satellite retrievals (e.g., MODIS, VIIRS) and in situ measurements. In situ airborne measurements performed at $1-\mathrm{Hz}$ frequency provide only low-resolution $(\sim 100 \mathrm{~m})$ samples, which do not allow us to resolve narrow zones of high gradients of microphysical variables. Recent high-resolution measurements provided important information of microphysical variables (Gerber et al. 2008; Katzwinkel et al. 2014; Schmeissner et al. 2015), but such measurements unavoidably face the inherent drawback of providing instantaneous $1 \mathrm{D}$ information about a dynamical, turbulent 3D field. For instance, despite decades of research, the existence of the undiluted core and its role in convective clouds, including boundary layer $\mathrm{Cu}$, remains a point of debate (Gerber 2000; Gerber et al. 2008; Romps and Kuang 2010a).

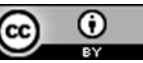

This article is licensed under a Creative Commons Attribution 4.0 license (http://creativecommons.org/ licenses/by/4.0/). 
The above arguments imply that the full picture for these cloud processes and properties should be completed with the help of numerical models. Large-eddy simulation (LES) models with grid spacing of tens to a few hundreds of meters were shown to provide important information about the macro properties of clouds (e.g., Heus et al. 2008; Heus and Jonker 2008; Siebesma et al. 2003; Khairoutdinov and Kogan 1999; Romps and Kuang 2010b; Dawe and Austin 2012; Khain et al. 2019; Heiblum et al. 2016b; Heiblum et al. 2016a). However, such grid spacing does not allow us to resolve zones of high gradients of microphysical and dynamical variables in the cloud. Some high-resolution studies were performed mostly using direct numerical simulations (DNS; Abma et al. 2013; Kumar et al. 2017; Mellado et al. 2018, and references therein). Very highresolution LESs with grid spacing of a few meters were used to study turbulent scale processes of entrainment and mixing at cloud tops of stratocumulus clouds (e.g., Stevens et al. 2005; Mellado et al. 2018) and trade wind cumulus (Hoffmann et al. 2014). Although most LES studies of trade cumulus clouds are done at $\sim 100$-m resolution, Seifert and Heus (2013) and Heus et al. (2009) studied their organization and tendencies to radiative-convective equilibrium state in simulations with grid spacing of $25 \mathrm{~m}$. However, the investigation of cloud microphysics was not the focus of any of the abovementioned studies, and therefore only simplified microphysical parameterizations were used to simulate droplet size distributions (DSDs). In contrast, the present study focuses on the relationship between the adiabatic core, mixing, and the microphysical processes that determine the evolution of DSDs in trade wind $\mathrm{Cu}$.

Cloud motions are driven by several processes that occur in many scales, and hence are quite complicated. Separating the dynamical and microphysical processes into moist adiabatic and those leading to deviation from adiabaticity (e.g., turbulent mixing and cloud-environment interactions) can simplify the analysis of cloud structure formation. As an example, in undiluted areas, one can approximate thermodynamic and microphysical profiles using simple adiabatic models, and evaluate liquid water content (LWC), number concentration $\left(N_{d}\right)$, relative humidity $(\mathrm{RH})$, effective radius $\left(r_{e}\right)$, and so on. These values can serve as local boundary conditions for the other regions in the cloud that are influenced by nonadiabatic processes like mixing. The deviation of the mixed regions from adiabaticity and separation of those regions can also be studied by considering the multiscale motions of the cloud as a combination of convective and turbulent motions (Pinsky et al. 2021a). The convective motions in the scale of the cloud would be linked to the adiabatic regions and their thermodynamic and microphysical properties. This view simplifies the analysis of cloud processes and their treatment in large-scale models.

Since deviation from adiabaticity highly depends on mixing, it affects the clouds' size, lifetime, and microphysical properties via the effect on DSDs. The effect of mixing on DSDs is one of the most important questions, since the DSD determines the cloud's radiative properties and initiation of rain (Khain and Pinsky 2018, ch. 5.10). Mixing of different cloudy volumes implies superposition of DSDs, which have modes in different drop sizes due to the different histories of the parcels, and leads to a wider multimodal DSD (Lasher-Trapp et al. 2005; Magaritz-Ronen et al. 2014). Mixing of a cloudy volume with its subsaturated environment acts to dilute the cloudy volume and evaporate droplets, either partially or completely. It decreases the droplets' number concentration $\left(N_{d}\right)$ and the liquid water content, and can also change the DSDs' shape and hence the characteristic droplet size (i.e., effective/mean/mean volume radius) and the DSD moments.

When sedimentation and radiation can be neglected, the level of adiabaticity (i.e., deviation from adiabatic values) can be a direct measure of mixing. Hence, we use adiabatic fraction (AF) as a measure of adiabaticity to study the existence of adiabatic regions in the cloud and the continued changes of DSD's properties from the cloud's undiluted core to cloud edge. $\mathrm{AF}$ is defined as $\mathrm{LWC} / \mathrm{LWC}_{\mathrm{ad}}$, where $\mathrm{LWC}_{\mathrm{ad}}$ is the value an adiabatic parcel would have when adiabatically lifted from the cloud base. Some observational studies of cumulus and stratocumulus clouds showed dependences of microphysical parameters, such as droplet effective radius and DSD width on AF (Freud et al. 2008; Pawlowska et al. 2006; Zhang et al. 2011). Previous studies used different approaches to calculate AF (Schmeissner et al. 2015; Pandithurai et al. 2012; Kim et al. 2008; Bera 2021; Zhang et al. 2011; Pawlowska et al. 2006; Freud et al. 2008; Gerber et al. 2008; Chandrakar et al. 2021); The method used to calculate AF in this study is discussed in Eytan et al. (2021). In that study, different derivations and approximations for calculating AF are tested by comparing them to a passive tracer, and it is shown that some of the approaches have higher sensitivity, and that not all of them are adequate for all shallow clouds.

The novel feature of the present study is the utilization of 10-m-grid-spacing LES with spectral bin microphysics (SBM) for investigation of microphysics and dynamics of boundary layer $\mathrm{Cu}$ during the growth and mature stages while using $\mathrm{AF}$ as the measure of adiabaticity. We simulate isolated nonprecipitating clouds of different sizes and aerosols concentrations, and analyze 3D fields of cloud variables. This may fill in some gaps between the partial information obtained by in situ measurements and results of simplified models and the realistic complex structure of the cloud and its controlling processes.

The main goals of this study are 1) to explore whether there are adiabatic regions in $\mathrm{Cu}$ clouds, 2) to quantify the dimension of the adiabatic region, as well as the diluted regions, and 3) to analyze the dependencies of cloud's properties (first of all, DSDs and their parameters) from the adiabatic core to the cloud's edge, using AF as a measure of adiabaticity/mixing.

\section{Methods of analysis}

\section{a. Model description}

LESs were performed using the System for Atmospheric Modeling (SAM; Khairoutdinov and Randall 2003; http:// rossby.msrc.sunysb.edu/ marat/SAM.html) coupled with the Hebrew University SBM scheme (Khain et al. 2004; Fan et al. 2009). DSDs and distributions of cloud condensational nuclei $(\mathrm{CCN})$ are given on a logarithmic mass grid containing 33 bins. The range of droplet radii was from $2 \mu \mathrm{m}$ to 3.4 
TABLE 1. The conditions of the simulations and the main parameters of the simulated clouds.

\begin{tabular}{lccccc}
\hline \hline $\begin{array}{c}\text { Type of simulated } \\
\text { clouds }\end{array}$ & $\begin{array}{c}\mathrm{CCN} \text { concentration } \\
\left(\mathrm{cm}^{-3}\right)\end{array}$ & $\begin{array}{c}\text { Initial radius of } \\
\text { temperature } \\
\text { perturbation }(\mathrm{m})\end{array}$ & $\begin{array}{c}\text { Maximal cloud-top } \\
\text { height }(\mathrm{km})\end{array}$ & $\begin{array}{c}\text { Maximal total mass } \\
\left(10^{5} \mathrm{~kg}\right)\end{array}$ & Cloud lifetime (min) \\
\hline Large & 500 & 500 & 2.11 & 2.15 & 38 \\
Clean & 50 & 500 & 2.03 & 2.23 & 30 \\
Medium & 500 & 250 & 1.83 & 0.34 & 18 \\
Small & 500 & 50 & 1.39 & 0.07 & 16.5 \\
\hline
\end{tabular}

$\mathrm{mm}$, and dry CCN maximum radius is $2 \mu \mathrm{m}$. Since the mass grid is logarithmic, the resolution is high for the smaller droplets within the size range $<20 \mu \mathrm{m}$. The diffusion growth/evaporation is calculated using a semi-analytical approach, when drop growth and the change in supersaturation are calculated by solving a coupled differential equation system, so droplet growth and the decrease in supersaturation are calculated at the same time. The method of drop mass change represents a semi-Lagrangian advection scheme of the first order that is stable at Courant numbers exceeding 1 . To decrease some diffusivity and DSD artificial broadening (Morrison et al. 2018), a method analogous to movable mass grid during diffusion growth (see also Kogan 1991), as well as modified remapping techniques (Khain et al. 2008), was used, which substantially reduced the numerical diffusivity. Comparison of the first radar echo altitudes simulated by the SBM with observations in $\mathrm{Cu}$ developing under different aerosol conditions, as well as comparison of the widths of DSDs at different heights, indicated a good agreement (e.g., Benmoshe et al. 2012; Khain et al. 2013). The high sensitivity of DSDs shapes to the aerosol concentration (e.g., Ilotoviz and Khain 2016; Benmoshe et al. 2012; also the present study, see below) showed that numerical broadening by diffusional growth is small compared with the broadening caused by natural processes in the cloud. The same conclusion was reached by Pardo et al. (2020). Collision-coalescence is solved by the stochastic collision equation with minimal diffusivity based on exponential flux method following Bott (1998). The collision kernels were calculated using an exact superposition method (considering the flow fields around colliding droplets) described by Pinsky et al. (2001). Activation of CCN to droplets, described in Khain et al. (2019), is based on the Köhler theory and uses the calculated values of supersaturation with respect to water and the critical $\mathrm{CCN}$ radius. The remaining $\mathrm{CCN}$ are advected with air motion, and can be activated in case of a certain increase in supersaturation. Drop sedimentation is calculated using fall velocities determined by Beard (1976).

\section{b. Simulation design}

Single $\mathrm{Cu}$ were simulated under the BOMEX thermodynamic conditions. The setup was taken from Siebesma et al. (2003), namely vertical profiles of water vapor mixing ratio and potential temperature with an inversion at 1500-2000 $\mathrm{m}$, large-scale forcing, and surface fluxes. The background wind was set to zero and the surface fluxes were kept constant. Single clouds were initiated by a perturbation of $0.1 \mathrm{~K}$ in the center of the domain with a varying horizontal radius of 500, 250, and $50 \mathrm{~m}$ for large, medium, and small clouds, respectively. The vertical size of the perturbation was kept at $100 \mathrm{~m}$ (from the surface) in all simulations. The perturbation decays to zero as a cosine square function of $0<x<$ $\pi / 2$, and random noise was added (Ovtchinnikov and Kogan 2000). Such temperature perturbation leads to clouds of realistic shapes with a large plate cloud base. The domain size was $5.12 \mathrm{~km}$, which is much larger than the largest cloud's width $(\sim 800 \mathrm{~m})$, to avoid effects of the cyclic boundary conditions. Spatial resolution was $10 \mathrm{~m}$ with 0.5 -s dynamical time steps. The vertical grid spacing was $10 \mathrm{~m}$ within the lowest 3-km layers (maximal cloud-top height is $2 \mathrm{~km}$ ) and increased to $50 \mathrm{~m}$ at the upper levels. The top boundary is located at $4 \mathrm{~km}$ with a sponge layer. For subgrid parameterization of turbulence, we used a 1.5-closure scheme (Khairoutdinov and Randall 2003). Following Jaenicke (1987, 405-420) and Altaratz et al. (2008) the size distribution of the aerosols was determined as the sum of three lognormal distributions describing fine, accumulation, and coarse aerosols modes. For simplification, we set aerosol concentration $\left(N_{a}\right)$ above cloud base $(\sim 600 \mathrm{~m})$ equal to zero, such that most aerosols enter the cloud from its base (some of the nonactivated CCNs ascend in the cloud and detrain to the interface zone and cloud surrounding by mixing). This highly reduces entrainment of new aerosols from the environment and eliminates activation at mixed regions of the cloud. The above simplification is done under the assumption that aerosol concentration decreases with altitude and that most cloud droplets origin from activation of aerosols entrained from the cloud base. This allows studying the basic effects of mixing and eliminates the effects of activation above cloud base of entrained aerosols (i.e., directly on the DSD and potential feedbacks on the dynamics of the cloud). Hoffmann et al. (2015) showed that during the growing stage (which is the stage studied in this work), lateral entrained aerosols contribute less than $15 \%$ to the total droplets concentration and that the significant role of such aerosols (more than $30 \%$ ) occurs during the dissipating stage. In this work, we simulated three clouds with $N_{a}$ of $500 \mathrm{~cm}^{-3}$, but of different sizes, as well as a clean cloud, similar in size to the largest one, but with $N_{a}=50 \mathrm{~cm}^{-3}$. The conditions of the simulations and the main parameters of simulated clouds are presented in Table 1. 

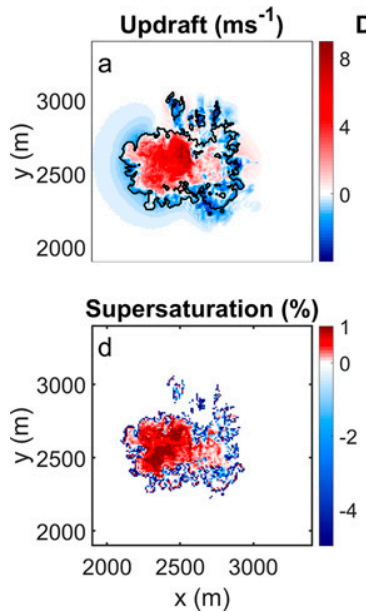
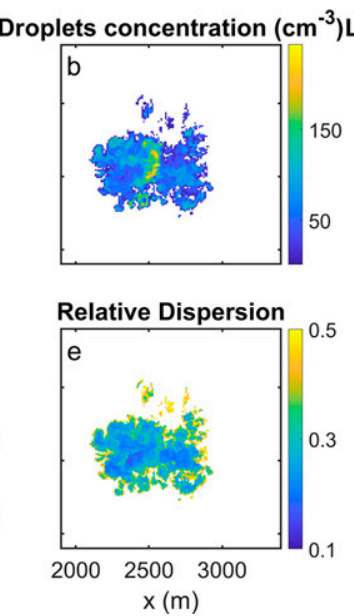
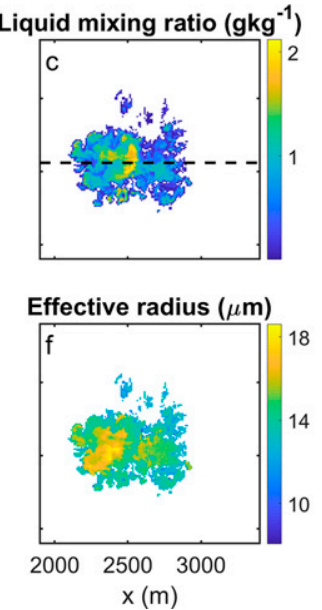

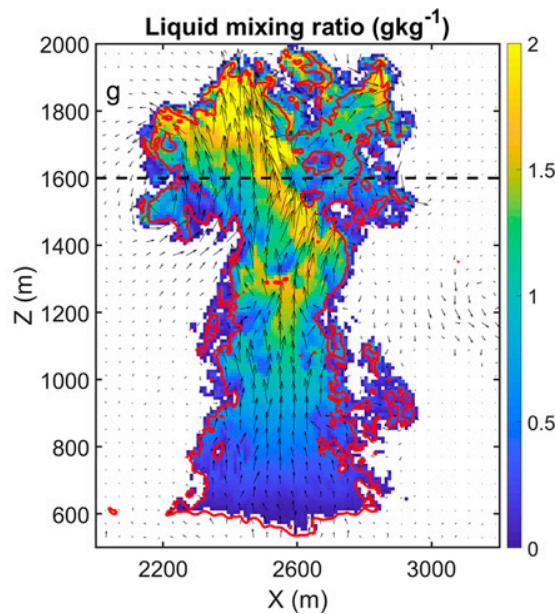

FIG. 1. Cross sections of the large cloud at the time of its maximal development (33 min; i.e., 13 min after cloud formation). Horizontal cross sections of (a) the vertical velocity at an altitude of $1600 \mathrm{~m}$ [marked by the dashed line in $(\mathrm{g})$ ], the black contour marks the cloud edges, where $q_{c}>0.01 \mathrm{~g} \mathrm{~kg}^{-1}$, (b) droplet concentration (noncloudy pixels are masked in white), (c) liquid water mixing ratio (the dashed line is the traverse shown in Figs. 2a-c), (d) supersaturation, (e) DSD relative dispersion, (f) effective droplet radius, and (g) vertical cross section of the cloud's liquid water mixing ratio at the center of the domain along the $y$ axis. The red contour marks supersaturation equals zero. Black arrows present the velocity components. Black dashed lines mark the altitude at which the horizontal cross sections are plotted in (a)-(f).

\section{Results}

\section{a. General features of the cloud structure}

The focus of this paper is the microphysical structure of $\mathrm{Cu}$ during the growth and mature stages, hence the clouds are presented mostly when they have reached their maximal development (maximal total mass of liquid water).

Figure 1 provides a general idea about macro- and microphysical structure of the large cloud at 13 min after its formation, when the cloud reaches maximum development (i.e., maximal total mass). Figures 1a-f present the horizontal cross section in the different fields at $z=1600 \mathrm{~m}$. Figure 1 a shows that maximum vertical velocity reaches $8 \mathrm{~m} \mathrm{~s}^{-1}$, and that the cloud updraft zone is surrounded by downdrafts. This is in agreement with the in situ measurements of Katzwinkel et al. (2014). Such a circulation near the cloud top is driven by a toroidal vortex (Zhao and Austin 2005; Houze 2014) that can be observed in the vertical cross section (Fig. 1g). The cloud core can be visually identified by the correlated location of strong updrafts $(w)$, high droplets concentration $\left(N_{d} ;\right.$ Fig. $\left.1 \mathrm{~b}\right)$, and high liquid water mixing ratio $\left(q_{c} ;\right.$ Fig. 1c). Figure $1 \mathrm{~d}$ shows the supersaturation field $(S)$. It can be seen that the periphery of the cloud is subsaturated, while the internal regions are supersaturated. The maximal supersaturation is located in the updraft zone and coincides with a zone of decreased droplet concentration $(x=2350 \mathrm{~m}, y=2500 \mathrm{~m})$. This region also exhibits the largest effective radius $\left(r_{e}\right.$; Fig. 1f) due to enhanced droplets growth (i.e., superadiabatic droplets). This occurs when a diluted parcel with low $N_{d}$ (due to an entrainment event) continues to ascend with the remaining droplets growing with lower competition (Baker et al. 1980; Lasher-Trapp et al. 2005; Yang et al. 2016). This phenomenon can be caused by the toroidal vortex (Eytan et al. 2021). We note that in this simulation there were no aerosols in the environment that could be entrained with the dry air and compete with the existing droplets. Broad DSDs near the cloud edges are presented in Fig. 1e as large relative dispersion values (defined as the ratio of the DSD width to the mean radius; $\varepsilon=\sigma / \bar{r})$. The maximum values of $r_{e}$ (Fig. 1f) occur both in the zone of maximum updraft, and in the zone of minimal droplet concentration and maximal $S$. These values exceed $14 \mu \mathrm{m}$, which is considered large enough for drizzle formation near the cloud top (Gerber 1996; Rosenfeld and Gutman 1994). Note that in this simulation, the drizzle amount $(r>40 \mu \mathrm{m})$ is negligible (smaller than $0.005 \mathrm{~g} \mathrm{~kg}^{-1}$ ), and is zero below $1500 \mathrm{~m}$. Despite the correlation of $r_{e}$ with $S$, its changes in the horizontal dimension are small, and range between 14 and $16 \mu \mathrm{m}$ at this level. Small horizontal changes of $r_{e}$ in convective clouds at any given altitude were found also in in situ measurements (e.g., Freud et al. 2008; Freud and Rosenfeld 2012; Prabha et al. 2011; Khain et al. 2013; Zhang et al. 2011; Konwar et al. 2021). Figure $1 \mathrm{~g}$ demonstrates the benefits of using such a high-resolution simulation, where detailed turbulent structures appear. The red contour in Fig. 1g marks the boundary of the saturated zone $(S=0)$ and shows the comparably thin subsaturated belt at the periphery of the cloud. One can also observe subsaturated regions of different sizes (largely small) of entrained environmental air volumes that can be modeled only by using such high resolution. A comprehensive presentation of the simulation results, including horizontal cross sections of the same variables from Fig. 1, and at the same time, is presented in the online supplemental material for the altitude of $1000 \mathrm{~m}$ (Fig. S1). There, we can observe a clear cloud structure with maximal values of $w, N_{d}$, and $q_{c}$ in the center of the cross section, and a 
Artificial aircraft trajectory
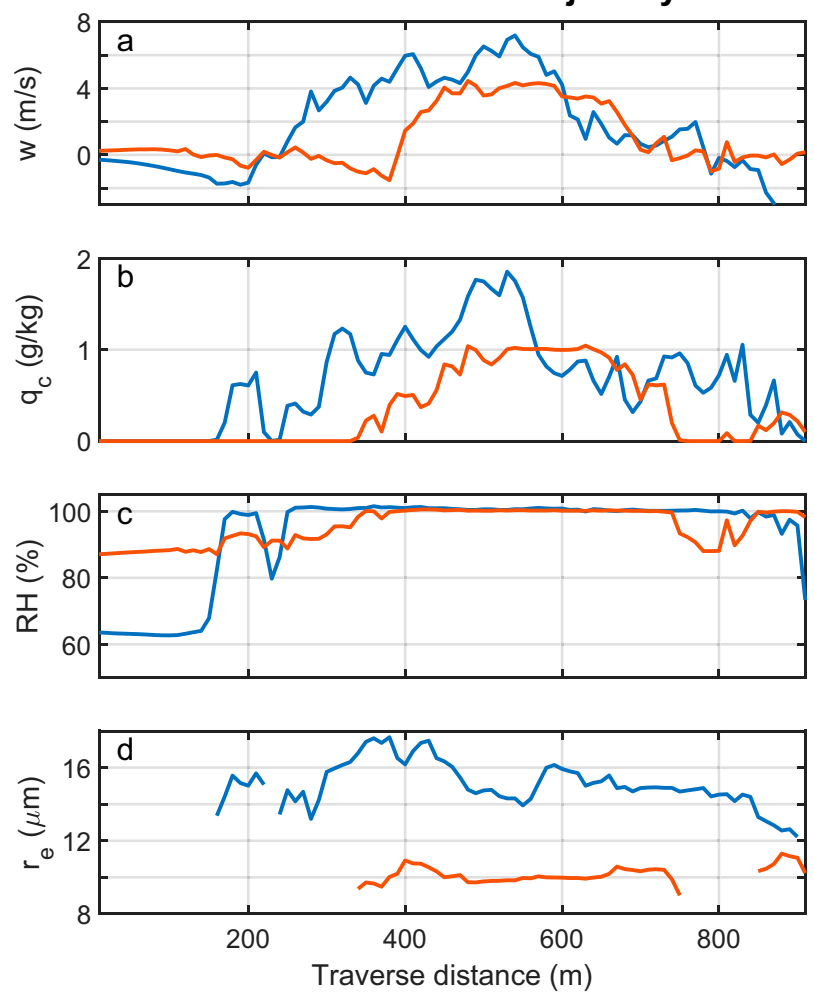

Mean values
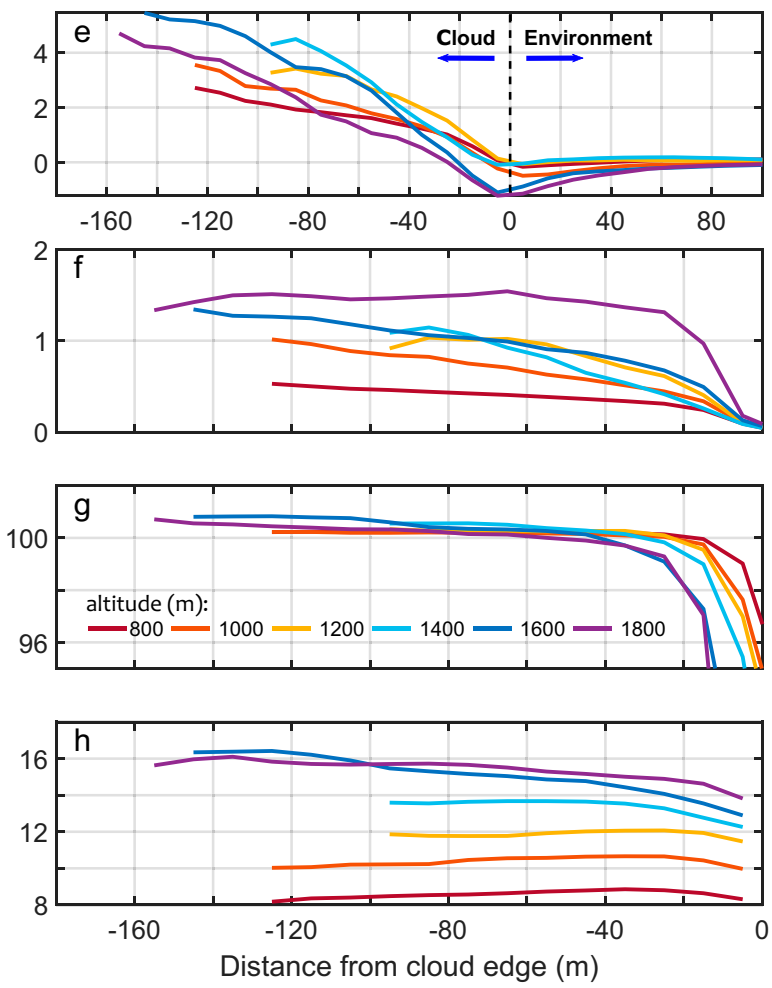

FIG. 2. Cloud variables as distance from cloud edge. (a)-(d) Artificial aircraft measurements for vertical wind, liquid mixing ratio, relative humidity (RH), and effective radius, respectively, at altitudes of 1000 and $1600 \mathrm{~m}$ (the latter is shown in Fig. 1c). (f)-(h) Mean values with respect to distance from cloud edge (DFCE) for several vertical levels (red and green curves mark the levels presented in the left panels). Note that (f)-(h) include only zones inside the cloud.

homogeneous distribution of the effective radius. We also observe buoyancy-driven downdrafts (subsiding shells) at 1000 $\mathrm{m}$, which are not related to the toroidal vortex that is found near the cloud top.

\section{b. Cloud parameters variability and the distance from the cloud edge}

If one wants to analyze horizontal profiles of different cloud properties, it is natural to observe them with respect to the distance from the cloud's edge or the core. This is useful, since such profiles can be compared with those obtained from in situ measurements, and it allows analyzing trends across the cloud-environment interface from both sides. The left panels of Fig. 2 mimic measurements along aircraft trajectories across the cloud, marked by the dashed lines in Figs. 1c and $1 \mathrm{~g}$, at $1600 \mathrm{~m}$. Note that the variability along the "trajectories" presents features that would be missed when using a coarser grid (as is commonly used in LESs or $1-\mathrm{Hz}$ measurements), such as the hole at $z=1000 \mathrm{~m}$ (see the part of $q_{c}=0 \mathrm{~g} \mathrm{~kg}^{-1}$ along the red line in Fig. 2b). Similar comparison of high-resolution simulations to measurements was conducted by Hoffmann et al. (2014). The right panels show the mean values of the same variables with respect to distance from the cloud's edge (DFCE) at several altitudes. The DFCE was calculated here as the Euclidian horizontal distance of each grid point from the closest cloudy voxel that had a "non-cloudy" neighbor. Voxels were determined as cloudy for $q_{c}>0.01 \mathrm{~g} \mathrm{~kg}^{-1}$. The data were binned and averaged at a bin width of $10 \mathrm{~m}$ for each layer, together with one layer below and one above it. DFCE bins that had less than 100 samples are not shown. The averaged values capture the important dynamical and microphysical features.

When averaged, $q_{c}$ shows a smooth decrease of the liquid water mixing ratio toward the cloud's edge, and a sharp decrease in the last $20-40 \mathrm{~m}$ near the cloud edge. Figures $2 \mathrm{a}$ and $2 \mathrm{~b}$ show a high correlation between the liquid water mixing ratio $q_{c}$ and $w$. The profiles of $w$ (Figs. 2a,e) display the subsiding shell that is discussed in Heus et al. (2008) and Katzwinkel et al. (2014), with the mean updraft decreasing toward the cloud edge, and even becoming negative a few tens of meters from the edge within the cloud. The horizontal gradients of buoyancy and absolute temperature are presented in Fig. S2, and support the concept of a buoyancydriven subsiding shell. A specific trajectory in Fig. 2c shows that RH can be less than $100 \%$ near cloud edges. Averaged $\mathrm{RH}$ values demonstrate that the region of sharp gradients in $q_{c}$ and $r_{e}$ is subsaturated, and extends to 20-40 m away from cloud edge (Fig. $2 \mathrm{~g}$ ). This region in the periphery of the cloud signifies a transition zone at the cloud's interface with the 

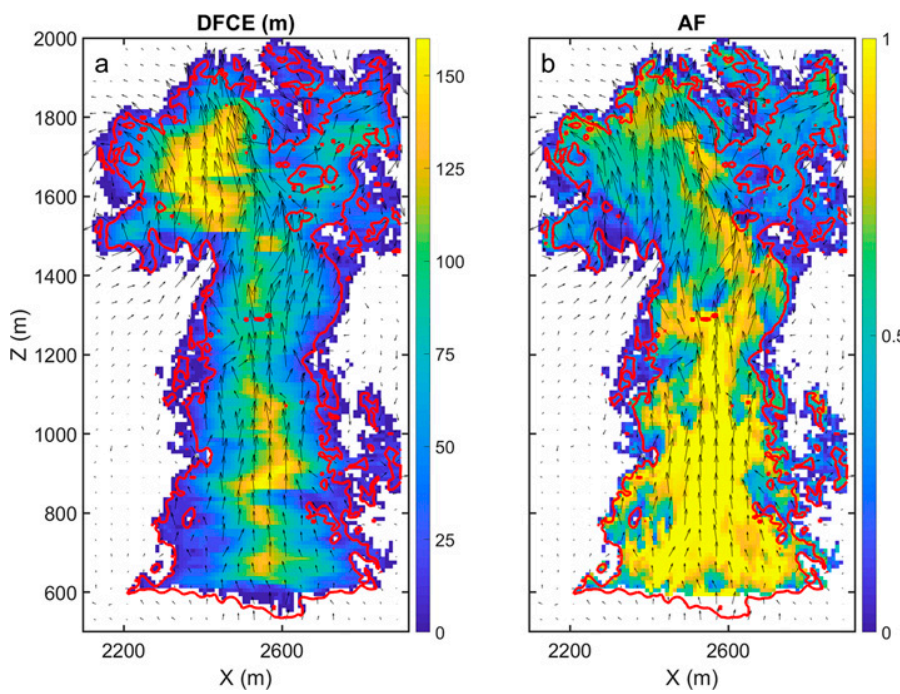

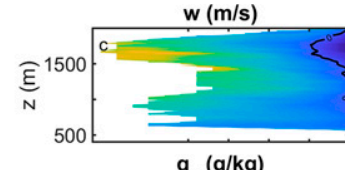

$q_{c}(g / k g)$

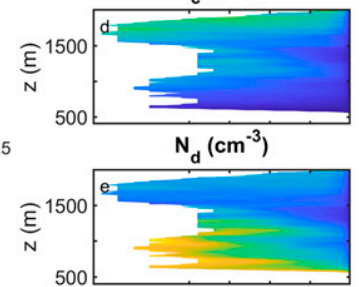

$B\left(\mathrm{~m} / \mathrm{s}^{2}\right)$

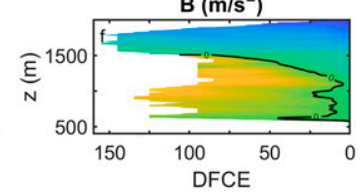

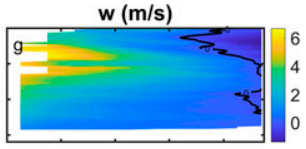

$q_{c}(g / k g)$

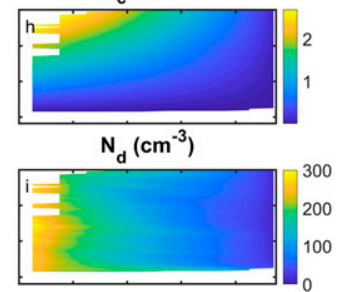

$B\left(\mathrm{~m} / \mathrm{s}^{2}\right)$

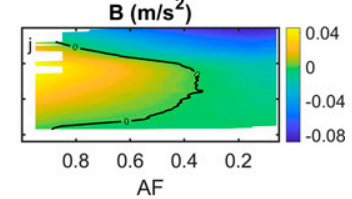

FIG. 3. Cloud variables in $z$-DFCE and $z$-AF spaces. (a) Vertical cross sections of the DFCE field. Black arrows represent the wind components, and the red contour corresponds to supersaturation equals zero. (b) As in (a), but for AF. (c) The mean vertical wind in a $z$-DFCE space. The black contour marks $w=0$. (d) As in (c), but for liquid mixing ratio. (e) As in (c), but for droplet concentration. (f) As in (c), but for buoyancy. The black line marks $B=0$. (g) As in (c), but in a $z$-AF space. (h) As in (d), but for AF. (i) As in (e), but for AF. (j) As in (f), but for AF.

environment. Similar behavior with a similar spatial scale can be also observed in high resolution in situ measurements by Gerber et al. (2008), Schmeissner et al. (2015), and Konwar et al. (2021). We note again that only high-resolution simulations can produce such a detailed description of variables, especially in the zone neighboring the cloud edge, referred to below as the transition zone.

After examining the cloud edges, we now turn to the other end point, the cloud core. Figure 3 shows different cloud variables in $z$-DFCE and $z-A F$ spaces. Figures $3 \mathrm{a}$ and $3 \mathrm{~b}$ are vertical cross sections of the cloud (similar to Fig. 1e), plotted for the DFCE and AF fields. Figure 3a depicts the spatial center of the cloud, located at maximum distances from the cloud edges, presented as the yellow vertical stripe of high DFCE. Figure $3 \mathrm{~b}$ shows the field of AF and the adiabatic core (where AF is close to unity). Note that the maximal values of the two parameters do not necessarily coincide, and that the mismatch is especially pronounced at the upper levels of the cloud. The zone of cloud core with high AF in Fig. 3 b resembles a structure of a turbulent jet (Houze 2014): the adiabatic core captures a large area of the horizontal cross section just above the cloud base, where above $1000 \mathrm{~m}$ it narrows, being affected by entraining eddies. The core appears to be continuous from the cloud base up to the inversion level (at $1500 \mathrm{~m}$ ). Around this level the core meandering increases and eventually breaks down to "small core filaments" when the cloud penetrates the inversion. Decrease in the scales of the undiluted cloud areas with height was reported in high-frequency measurements along linear aircraft tracks by Gerber (2000). AF shows significant variability within the $100 \mathrm{~m}$ above cloud base. This variability is caused by the inhomogeneity of the cloud base level and the sensitivity of AF to two small numbers: LWC and $\mathrm{LWC}_{\mathrm{ad}}$ (Eytan et al. 2021).

Figures $3 \mathrm{c}-\mathrm{f}$ show the fields of average values of $w, N_{d}, q_{c}$, and buoyancy $(B)$ in a $z$-DFCE space, and Figs. $3 g-j$ depict the same variables on a $z-\mathrm{AF}$ space. The averaging was performed by binning the data in each layer according to DFCE (or AF), with bin widths of $10 \mathrm{~m}$ (or AF increment of 0.1 ). Comparison of averaged cloud variables in $z$-DFCE and $z$-AF spaces show a general agreement between the fields: the increase in the distance from the cloud edge and increase in AF both indicate increase in vertical velocity, LWC, $N_{d}$, and buoyancy. However, AF seems to be a more convenient measure for separation between the clouds' core and margin properties. While the $q_{c}$ field serves as a sanity check and shows an almost linear increase in both the vertical and "coreward" directions, the maximal $w$ and $N_{d}$ are found in the adiabatic core (high AF values). Figures $3 f$ and $3 \mathrm{j}$ present the clouds' buoyancy, which is maximal in the undiluted core because of the higher temperature and water vapor content. One can see that $B>0$ at $\mathrm{AF}>0.4$, while at cloud edges, where AF is low, $B<0$ (which drives the subsiding shells). Above the stable inversion layer (at an altitude of $1500 \mathrm{~m}$ ) the buoyancy is negative. The black contour in Figs. $3 \mathrm{f}$ and $3 \mathrm{j}$ marks the zero buoyancy line. This line is much smoother in the case of AF than DFCE, further corroborating the argument above. Averaging the cloud parameters according to DFCE leads to grouping of cloudy volumes with different histories that have gone through various mixing processes, and thus the $z$-DFCE maps (Figs. 3c-f) are noisier. This claim is also supported by noting that the $z$-AF maps show higher (lower) maximal (minimal) values of each parameter; sorting 

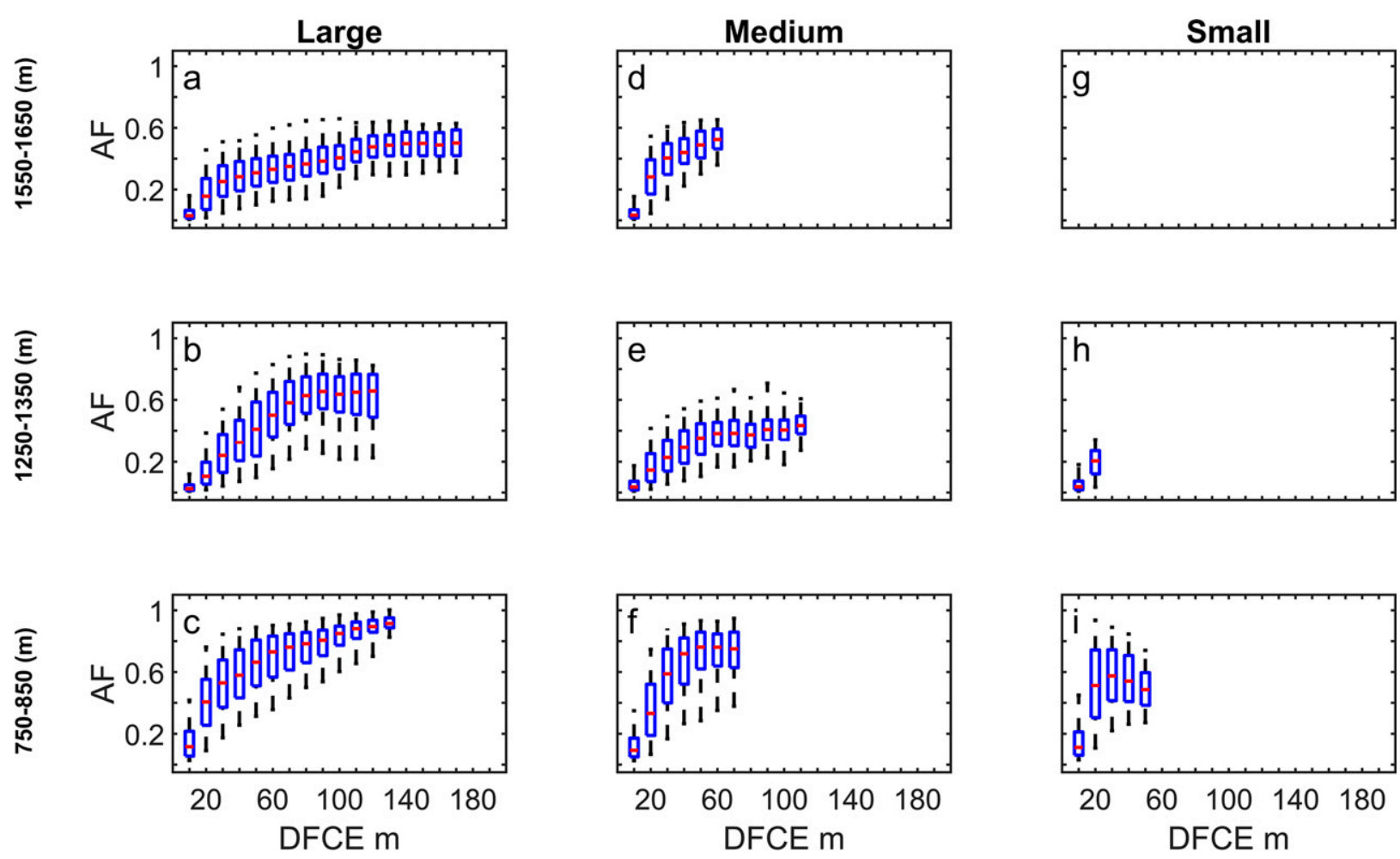

FIG. 4. AF vs DFCE for clouds of different sizes. Each cloud was analyzed at the time of its maximal development, and the distributions of AF values in DFCE bins are represented by boxplots for different clouds (columns) and altitudes (rows). The red line in each boxplot marks the median; the higher and lower edges of the boxes represent the 75th and 25th percentiles, respectively, and the dashed error bars mark the 90th and 10th percentiles. Results are shown for (a)-(c) large, (d)-(f) medium, and (g)-(i) small clouds, all with $N_{a}=500 \mathrm{~cm}^{-3}$.

according to AF groups together pure core or transition zone parcels, thus providing higher or lower average values (e.g., there are less outliers of diluted volumes in the core). Moreover, it does not depend as strongly on the cloud edge geometry.

The relations between AF and DFCE for clouds of different sizes are shown in Fig. 4. The AF field of each cloud was binned in each layer according to the DFCE (in bin widths of $10 \mathrm{~m}$ ), and the boxplot shows the median value for each bin (red line) and the spread of the data. The common relations of AF and DFCE for all clouds are such that AF increases deeper in the cloud (high DFCE) until it saturates at some value in the farthest distances; thus, the gradient and the saturation values of AF characterize the entrainment/mixing process of the cloud's parcels at a certain altitude. The variability of the AF values inside each DFCE bin also provides important information about the properties of the mixing. As an example, Fig. 4c (left column) shows that near the cloud base of large clouds AF increases quickly, reaching values close to unity. The low variability of AF deep inside the cloud (largest DFCE bins) indicates that the core is located in the center of the cloud, where there are only adiabatic volumes. Higher in the cloud (Figs. 4a,b), we can see that the median values saturate at smaller distances from the cloud edges, and that the median $\mathrm{AF}$ values plateau at lower $\mathrm{AF}$ (i.e., around $\mathrm{AF}=$ 0.6 and $\mathrm{AF}<0.6$ for Figs. $4 \mathrm{~b}$ and $4 \mathrm{a}$, respectively). We note that above the inversion, despite the low AF values of the 90th percentile, high AF values exist also at higher levels and for a broad range of distances, as can be seen in Fig. 3, and is shown next. A comparison of the dependencies of AF on DFCE for the large cloud and the smaller ones shows that all of the arguments above are valid for all cloud sizes. The gradient and the saturation values decrease for smaller clouds, as can be seen when comparing the different panels in the same row. The small cloud seems to be highly diluted, having a weak relation of AF to DCFE even near the cloud base (Fig. 4i). Figure $4 \mathrm{~g}$ is empty because the small cloud did not reach this level.

Figure 5 presents fields of $w, q_{c}, N_{d}$, and $B$ in the $z-\mathrm{AF}$ space for the different clouds. Comparison of the large, medium, and small clouds shows that dilution by entrainment and mixing propagates inside toward the core faster in the smaller clouds. This is demonstrated by the lower values in the core of all four parameters for smaller clouds, and by the faster decrease in number of volumes with high AF (shown as missing data of high AF at higher levels). We see, therefore, that smaller (and thinner) clouds dissipate faster, having shorter lifetimes (see Table 1).

\section{c. Occurrence and locations of adiabatic regions}

The previous sections demonstrated the usefulness of using $\mathrm{AF}$ as a continuous metric for mixing/dilution. In this section, 

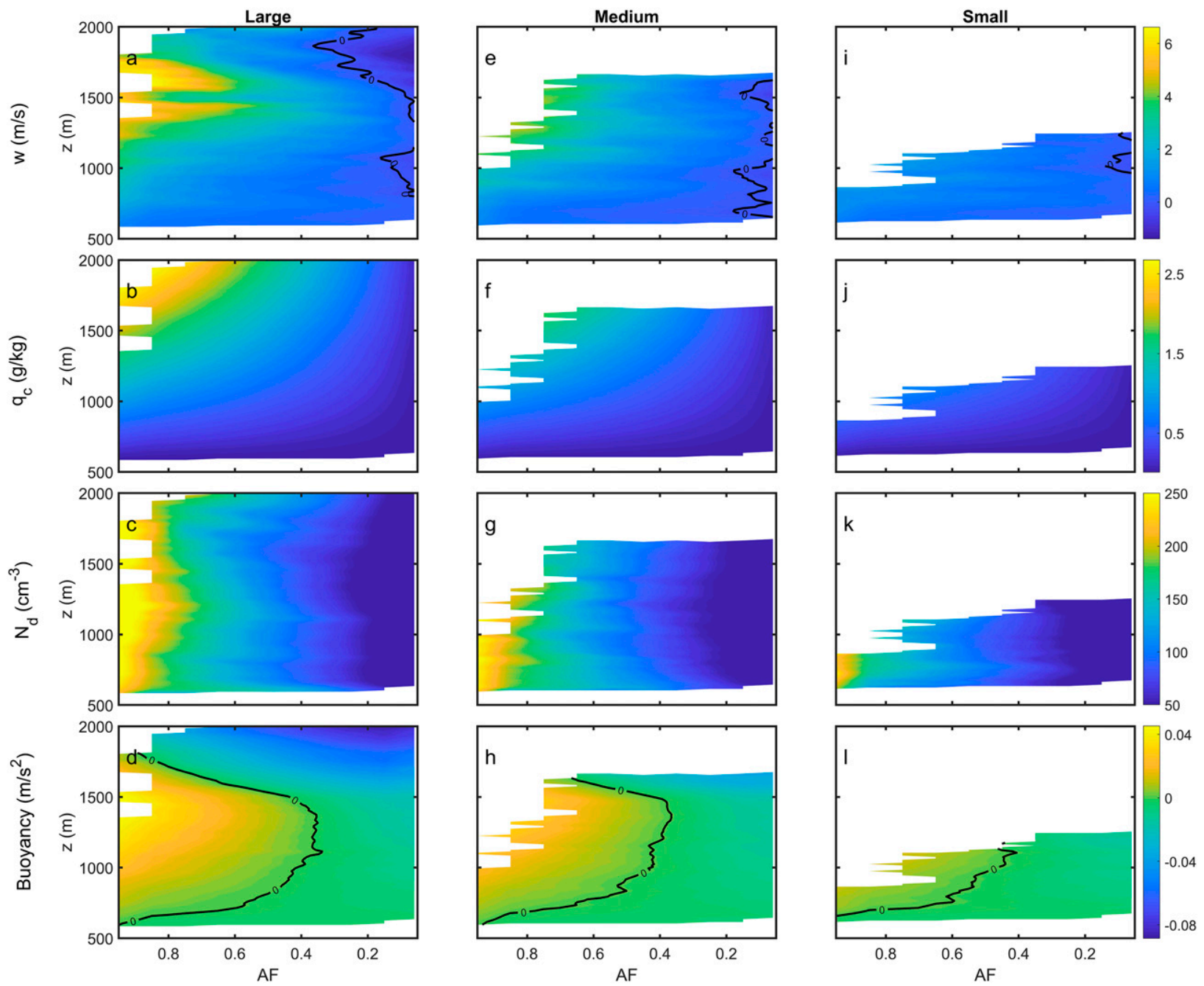

FIG. 5. The $z-\mathrm{AF}$ space for clouds of different sizes. The fields are shown at the time of maximum cloud development. The fields of (top to bottom) $w, q_{c}, N_{d}$, and $B$ were binned and averaged for each altitude, according to AF, with increment widths of 0.1 .

we analyze the occurrence of different values of AF in different clouds, and the AF dependence on altitude. Figure 6 shows the $2 \mathrm{D}$ probability distribution (occurrence) of AF at different altitudes for the large, medium, and small clouds at the time instance of maximal cloud development. It can be seen that in the lower parts of the cloud most of the volume can be considered as core with high AF (dominated by adiabatic processes), while in the higher levels most volumes are
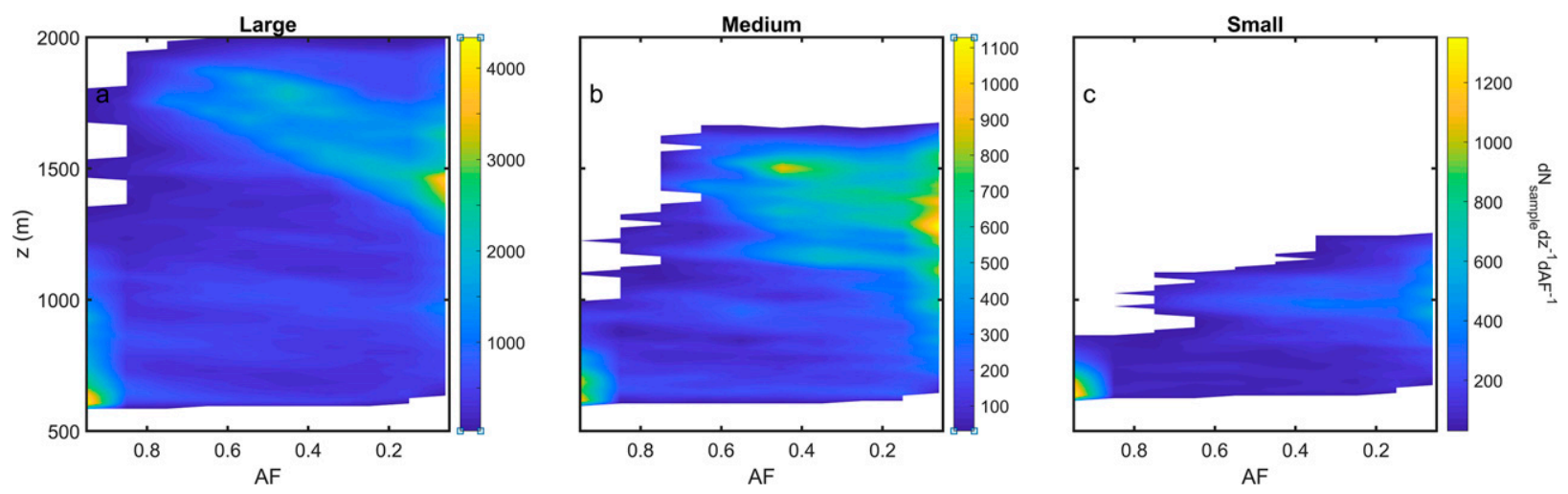

FIG. 6. The 2D probability distribution of AF with altitude: (a) large cloud, (b) medium cloud, and (c) small cloud. 

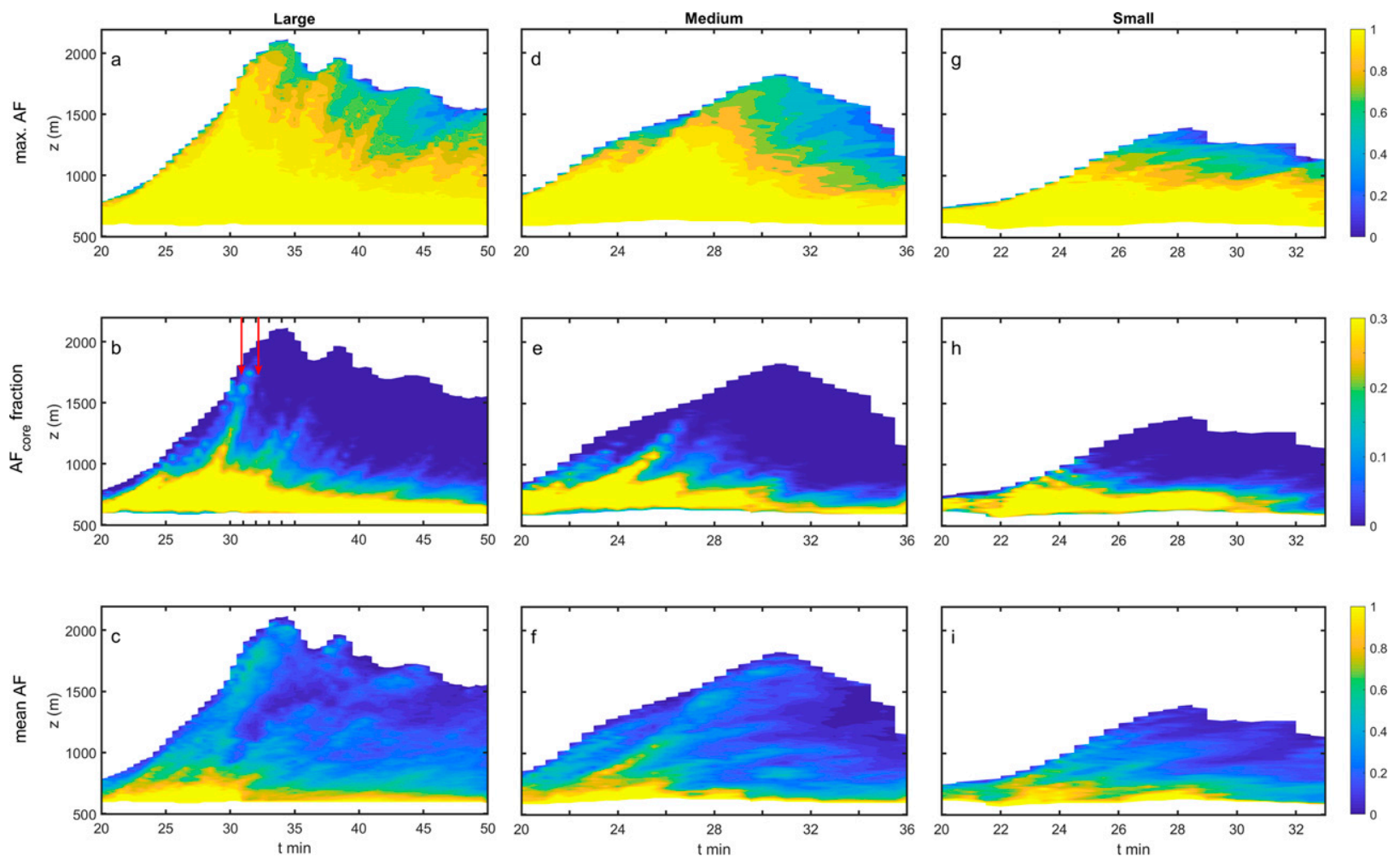

FIG. 7. The $z$-time AF diagrams. (top) Maximal AF. (middle) Area fraction of the core, defined as the area of regions of AF $>0.9$ normalized by the cloud horizontal cross section. (bottom) Mean AF. The different time axes in each column are due to the different lifetimes of the simulated clouds. The space between the red arrows [in (b)] marks the lifetime of an adiabatic core that captures more than $10 \%$ of the cloud area.

sub-adiabatic or part of the transition zone $(\mathrm{AF}<0.2)$. The location of the transition zone on the AF axis will be presented in the next section.

Decrease in the occurrence of high AF with altitude reflects a decrease in the size of the adiabatic core. On the other hand, the occurrence of very low AF values increases. The highest occurrence frequency of low AF values takes place at an altitude of $\sim 1400-1500 \mathrm{~m}$, where a high entrainment rate is caused by the toroidal vortex (i.e., ring vortex; see examples in the large cloud in Figs. 1 and 3). This is true also for the medium clouds. The small cloud is entrained in almost all levels, as can be seen in Fig. 6c, hence, its core depth is only 150-200 m. The high occurrence of high AF only near cloud base can lead to an assumption that the cloud is dominated by adiabatic processes in the lower levels, and only by mixing in the higher levels. However, this is not the case, since the cloud core exists up to the inversion layer, and small volumes of high AF occur up to the cloud top. Thus, volumes of high AF can be a source of cloud droplets and energy through most of the cloud column, i.e., adiabatic processes in the core contribute to the structure of all other sub-adiabatic volumes in similar altitudes.

Figure 7 shows $z$-time diagrams for all clouds during their life cycle. The diagrams are plotted for maximum AF, adiabatic core area fraction (where AF $>0.9$ ), and horizontally averaged AF. One can see three stages of cloud evolution: a development stage with increase of the cloud-top height (e.g., until $33 \mathrm{~min}$. for the large cloud), a short mature stage, and a comparatively slow cloud decay stage, accompanied by a decrease in the cloud top height. During the growth stage the adiabatic core (even if small) exists at least for half of the cloud's depth (up to $z=1800 \mathrm{~m}$ for large clouds; Fig. 7b), and volumes with high AF are found almost up to the cloud top (reach $2000 \mathrm{~m}$ for large clouds; Fig. 7a). The first two rows indicate the existence of small adiabatic volumes in the highest levels of the cloud. Note that undiluted zones of high AF are zones of maximum updrafts and buoyancy, which determine the maximum cloud-top height. Those volumes, which might be considered unimportant can determine the cloud top, and also be a part of a mechanism that transports large droplets to the cloud top, which can lead to rain initiation. The averaged values of AF near the cloud top are approximately $0.4-0.6$ at the developing stage and $0.1-0.3$ at the decaying stage (Fig. 7b), which is in good agreement with high-frequency measurements in $\mathrm{Cu}$ (Schmeissner et al. 2015). The figure shows that an adiabatic cloud core with an area fraction exceeding 0.1 exists only for a short period of $\sim 1$ min above the inversion (see the space between the arrows in Fig. 7b). Since the cloud exists in those altitudes for $\sim 10$ min, it gives a probability of only $1 \%$ to measure an adiabatic volume (not considering the trajectory of the aircraft). This might explain why the adiabatic core was not detected in many in situ measurements. 

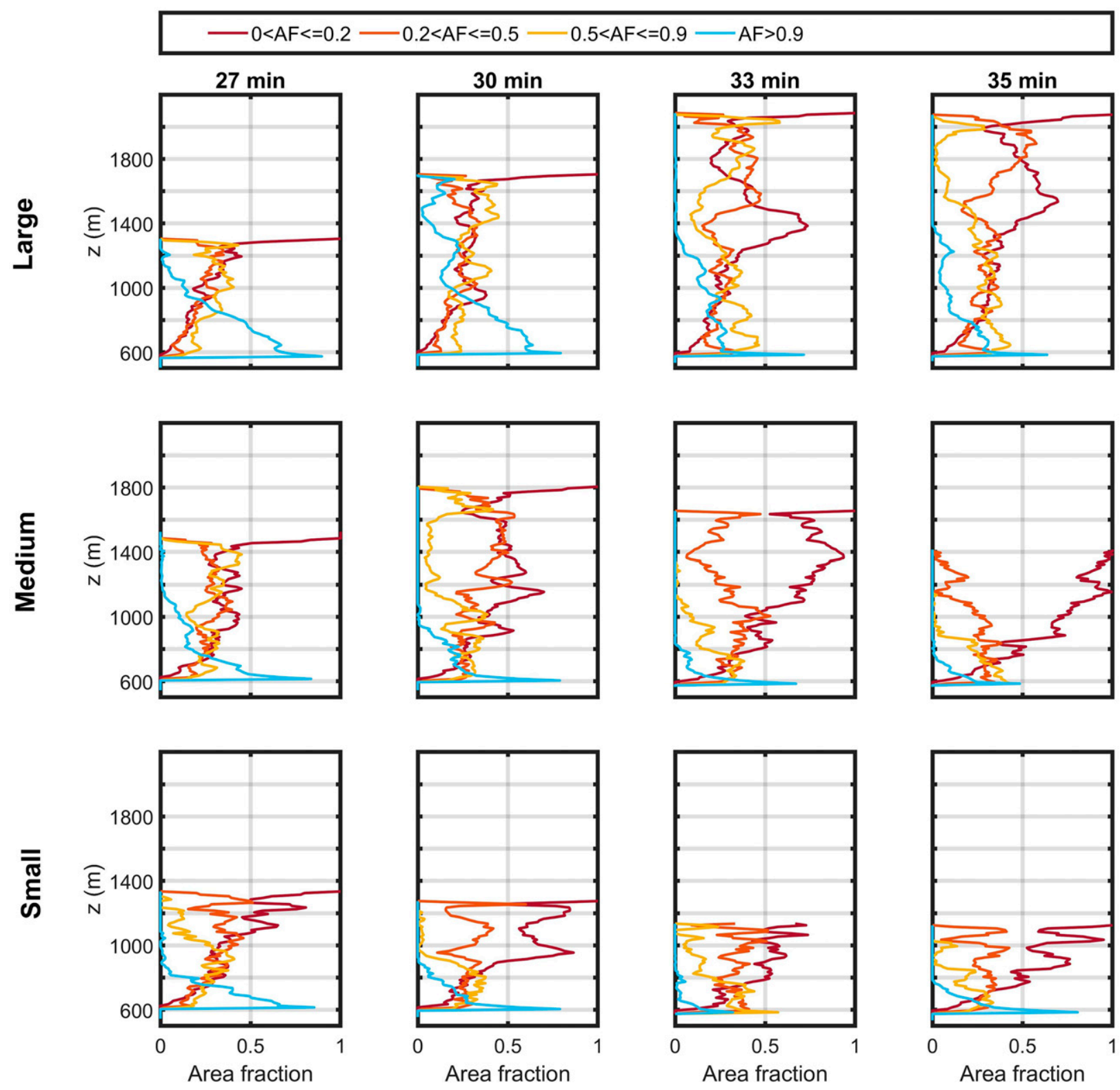

FIG. 8. Vertical profiles of area fractions for four AF ranges: core ( $\mathrm{AF}>0.9$; blue), slightly diluted $(0.5<\mathrm{AF} \leq 0.9$; yellow), diluted ( 0.2 $<\mathrm{AF} \leq 0.5$; orange $)$, and transition zone $(\mathrm{AF} \leq 0.2$; red) for different clouds at different time instances.

Figure 8 shows the vertical profiles of area fraction zones for four ranges of $\mathrm{AF}$ : core (AF $>0.9$; blue), slightly diluted $(0.5<\mathrm{AF} \leq 0.9 ;$ yellow $)$, diluted $(0.2<\mathrm{AF} \leq 0.5$; orange $)$, and transition zone ( $\mathrm{AF} \leq 0.2$; red).

Several important features can be observed in Fig. 8. First, as discussed above, the adiabatic core exists in all clouds, but the area fraction of the core decreases with height. This decrease, which is faster for smaller clouds, is compensated by the increase in the sub-adiabatic area with height. During the growth stage, the area fraction of all three sub-adiabatic regimes is very similar. At the decaying stage, which starts faster for the medium and small $\mathrm{Cu}$, the slightly diluted area fraction decreases, and the diluted area fraction increase. This separation is especially pronounced in the upper part of the clouds, and accelerates once the clouds cross the inversion. While the larger cloud contains an adiabatic core up to the inversion, the smaller ones lose their core below it, depending on their size. It is demonstrated how all three clouds are mostly adiabatic in the beginning of the life cycle, and then start to dissipate with time, at higher levels.

\section{d. The variability of DSD shapes in different $A F$ regimes}

One of the main goals of the study is to determine whether DSD shapes and parameters can be classified according to 

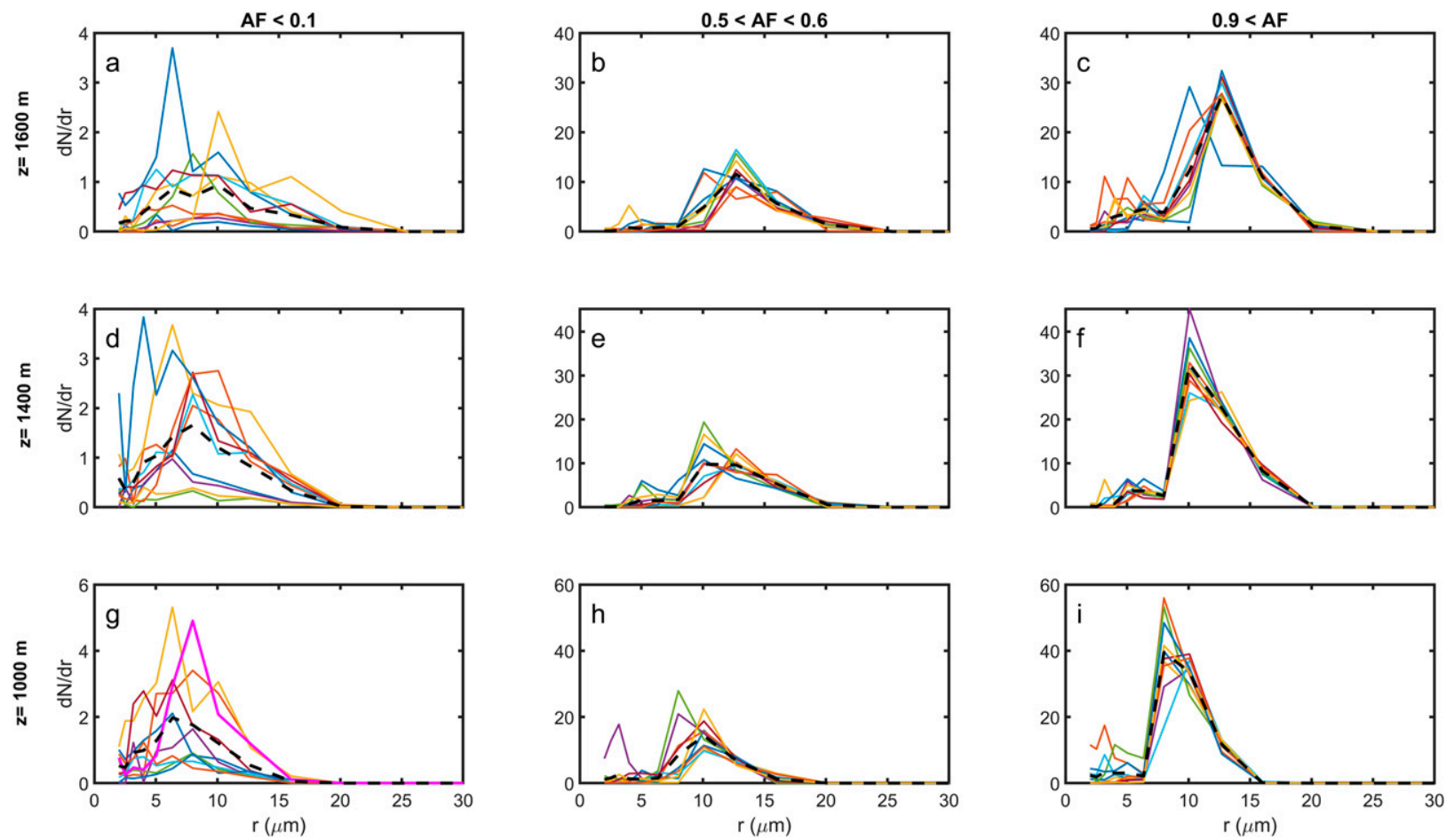

FIG. 9. Example of DSDs at different heights for different AF ranges for polluted cloud at $t=33$ min. Each panel shows 10 randomly chosen DSDs in different AF values at three altitudes (1000, 1400, and $1600 \mathrm{~m})$. Results are shown for (left) low AF $(0.1<\mathrm{AF}<0.2)$, (center) intermediate $\mathrm{AF}$ values $(0.5<\mathrm{AF}<0.6)$, and (right) high $\mathrm{AF}$ values $(\mathrm{AF}>0.9)$. Black dashed line is the mean DSD of all 10 samples.

AF. To achieve this goal, the cloud was classified according to altitudes and values of AF. DSDs from several randomly chosen grid points with AF belonging to the same range were plotted. A clean cloud developing at low aerosol concentration was simulated in order to investigate these relations under different aerosol conditions (see Table 1). The clean cloud is analyzed at its growth stage, before significant droplet sedimentation. This allows comparing the adiabaticity of polluted versus clean clouds (see Eytan et al. 2021). Figure 9 presents several examples of DSDs from the polluted (large) cloud at $t=33 \mathrm{~min}$. The examples are shown for three AF ranges-low $(0.1<\mathrm{AF}<0.2)$, intermediate $(0.5<\mathrm{AF}<$ $0.6)$, and high AF $(0.9<\mathrm{AF})$ - and for three different altitudes: $1000 \mathrm{~m}, 1400 \mathrm{~m}$ (below inversion), and $1600 \mathrm{~m}$ (above the inversion). Each panel contains 10 DSDs per one altitude and AF range. The average DSDs are also presented. The simulated DSDs show remarkable similarity to DSDs measured at a high resolution of $25 \mathrm{~Hz}$ by Konwar et al. (2021) in growing convective clouds over India, with similar droplet concentrations (see Fig. S3).

The observed similarity for the DSDs of the cloud core is due to their common and simple history of adiabatic ascent. This agrees with the adiabatic parcel theory: their modal droplet size increases with height, with the main DSDs mode centered at $r=8-12 \mu \mathrm{m}$. Unlike the classical parcel theory, the DSDs are broadening with height due to small secondary peaks (modes) at the smaller drop sizes $(r<5 \mu \mathrm{m})$. These small droplets arise as a result of in-cloud nucleation and a minor contribution from mixing (see section 3d). As AF decreases, the total droplet concentration decreases (note the DSD values at the $y$ axis) and the DSD shape variability increases (middle and left columns in Fig. 9). In those regimes, many shapes of multimodal DSDs can be observed, as a consequence of mixing (i.e., superposition of DSDs that experienced different histories). There are also examples of unique cases of DSDs that resemble the shape of the DSD in high AF, but decreased by a scale factor (e.g., the magenta curve in Fig. 9g). This could be the consequence of an adiabatic parcel experiencing only dilution caused by mixing scenarios that does not affect the DSD shape (e.g., inhomogeneous mixing, or mixing with volumes with relative humidity close to $100 \%$ ). Figure 10 is similar to Fig. 9, but for the case of a clean cloud. The DSDs in the clean cloud's core are similar. As expected, the mean drop size is larger than in the polluted case, and the maximal droplet sizes are twice as large as in polluted clouds, indicating substantial contribution of collisions. The secondary peak in the DSDs of regions of high AF is negligible compared to the polluted cloud in both absolute concentration, and relative to the main mode. This is because all aerosols were already activated in the cloud base for the clean case. The trend of larger variability of shapes with decreasing AF is maintained, although there is a lower number of multimodal DSDs in regions of low AF compared with the polluted cloud. 

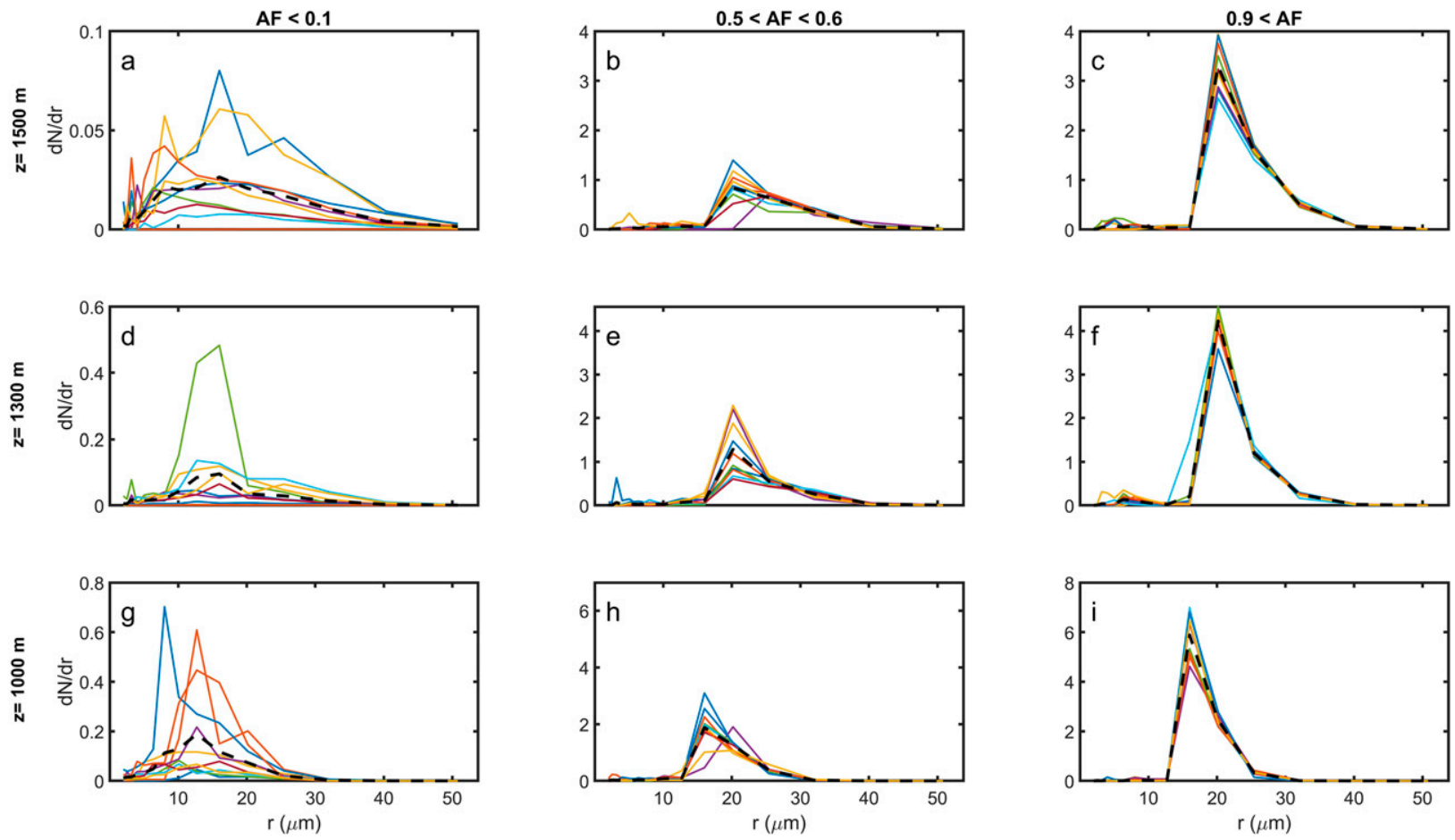

FIG. 10. As in Fig. 9, but for a clean cloud.

\section{e. Classification of DSD parameters with respect to $A F$}

Next, we examine the microphysical processes leading to the DSDs shown in Figs. 9 and 10. Figure 11 presents the rates of collision-coalescence (top row), droplets activation (bottom row), and supersaturation (middle row) for polluted and clean clouds. The top row in Fig. 11 shows that the peak in collision-coalescence occurs in the highest AF zone, near the cloud top, for both clouds. These are the locations of the largest drops (Fig. 2h) and highest liquid water mixing ratios (maximal $q_{c}$, Fig. 5, second row). This finding supports those reached by Khain et al. (2013), and leads to the conclusion that first raindrops (drizzle) form in the cloud core at the cloud top. This again stresses the importance of convective scale moist-adiabatic processes in cloud microphysics. The middle row in Fig. 11 shows that both clouds are subsaturated on average when $\mathrm{AF}<0.2$. This consistent feature, which was also observed for the smaller polluted clouds, marks the transition zone at the cloud edge, which was presented in Fig. 2 as the subsaturated regions in distance of $20-40 \mathrm{~m}$ from the cloud edge. We note that although it extends over a fifth of the axis on the AF space, this is a thin region located near the edge of the cloud and around entrained eddies before their dissipation; nevertheless, it occupies a considerable fraction of the cloud area, as shown in Fig. 8. The black contours in the middle row are for $S=0$, and are used to mark the transition zone in Fig. 12. The bottom row in Fig. 11 shows the droplets' activation rate within the cloud, with a clear peak near the cloud base (note that the color bar is saturated). At higher altitudes, in the core (high AF) of the polluted cloud, there is another moderate peak of secondary activation, marked by magenta contours at a value of $0.4 \mathrm{~cm}^{-3} \mathrm{~s}^{-1}$ (Fig. 11c). This activation leads to the formation of bimodal DSDs in polluted large $\mathrm{Cu}$ (see Fig. 9). This does not occur in the clean cloud, because all aerosols were activated in the cloud base due to their low concentration and high $S$ (Fig. 11f).

Figure 12 shows several microphysical parameters characterizing DSDs in the two clouds in the $z$-AF space: the mean relative dispersion $(\varepsilon)$, the ratio of effective radius to mean volume radius, number of the DSD modes, and the DSD skewness. Note that $\varepsilon$ changes with altitude only slightly, and ranges from 0.25 to 0.4 for the polluted cloud, and up to 0.55 for the clean cloud. Similar values of $\varepsilon$ were found in simulations of the medium and small size $\mathrm{Cu}$ (not shown). The results agree well with in situ measurements reported for shallow $\mathrm{Cu}$ (Tas et al. 2015; Liu et al. 2006), as well as for growing nonprecipitating convective clouds (Prabha et al. 2011; Khain et al. 2013). The weak dependence of $\varepsilon$ on height indicates DSD broadening with height (since the mean radius increases), which is a well-established feature of $\mathrm{Cu}$. Since adiabatic parcel solutions dictate that the DSD should become narrower with altitude (due to condensation alone), these observations were at the heart of a long-lasting paradox. The broadening was explained by several physical reasons that were suggested: in-cloud nucleation of small aerosols in zones of elevated supersaturation (Khain et al. 2000; Pinsky and Khain 2002) and collision-coalescence at the stage of diffusion growth (Pinsky and Khain 2002), whose rate can be accelerated by turbulence (Khain and Pinsky 2018; Wyszogrodzki et al. 2013). Wide in situ measured DSDs were also explained by the spatial averaging of the low-frequency 

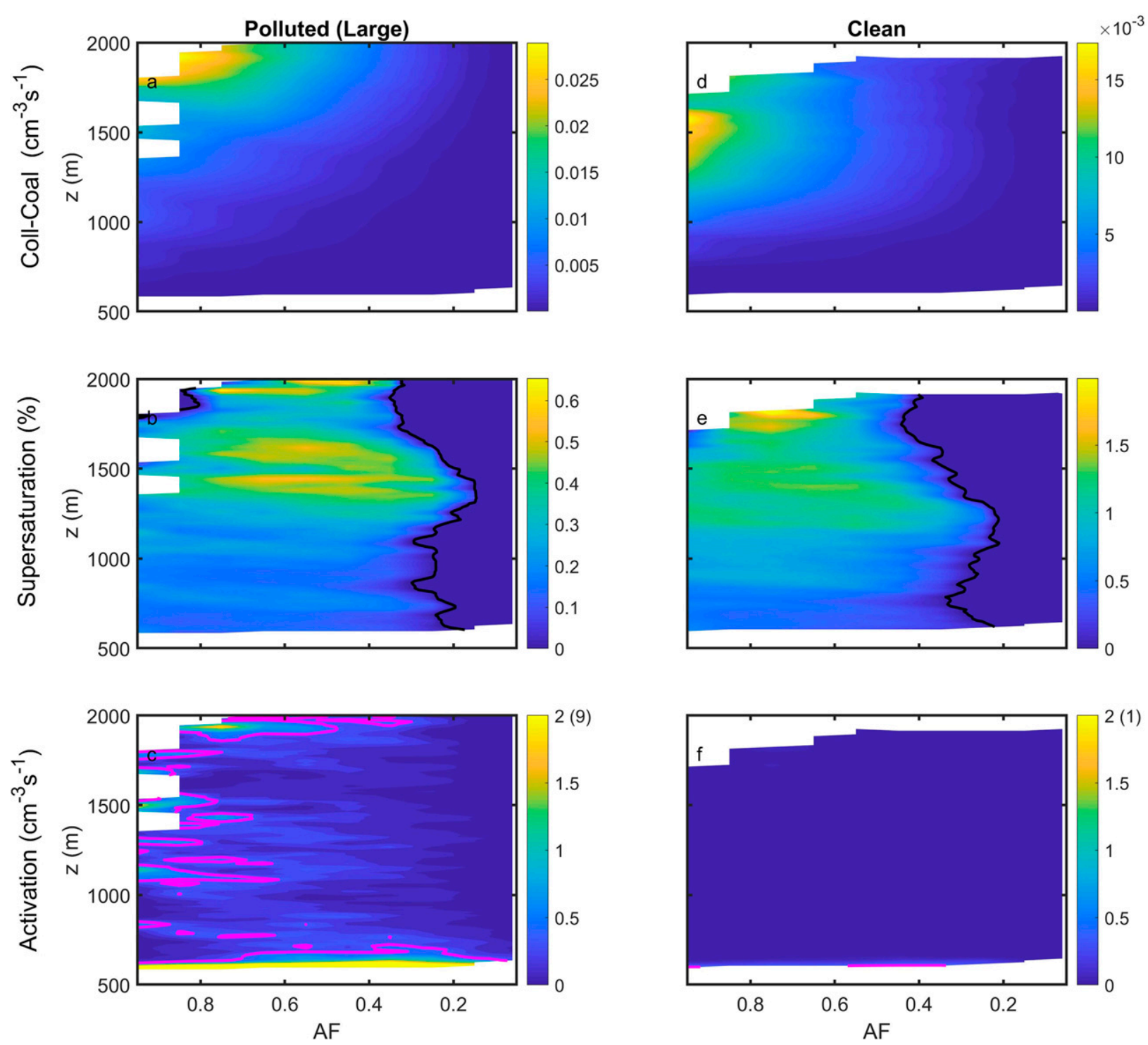

FIG. 11. Rates of the microphysical processes. Plots of $z$-AF space for different clouds (columns) and different microphysical processes as a function of $\mathrm{AF}$ (rows). (top) Collision-coalescence rate $\left(\mathrm{cm}^{-3} \mathrm{~s}^{-1}\right)$. (middle) As in the top row, but for supersaturation, also indicating zones of condensation/evaporation. Subsaturated values are not shown, and $S=0$ is marked by a black contour that bounds the transition zone. (bottom) As in the top row, but for droplets' activation rate $\left(\mathrm{cm}^{-3} \mathrm{~s}^{-1}\right)$. Magenta contours mark the region of secondary nucleation $\left(0.4 \mathrm{~cm}^{-3} \mathrm{~s}^{-1}\right)$.

measurements along the airplane track (Levin et al. 1996; Korolev 1994). Our high-resolution results and their resemblance to the high-frequency measurements (Konwar et al. 2021; Fig. S3) show that the effect of averaging is not the main cause of large DSD width.

In the horizontal dimension, the $\varepsilon$ in both clouds is the smallest in the cloud core (high AF) and increases toward the edge, since the DSDs in the vicinity of the cloud edge are wide and the mean radius is small. The black contours from Fig. 11 mark the subsaturated transition zone, where mixing and evaporation are dominant processes. The higher variability of DSD shapes in the transition zone means that mixing acts to superimpose DSD with modes in different sizes, resulting in the formation of bimodal and multimodal wide DSDs (Lasher-Trapp et al. 2005). In the clean cloud, $\varepsilon$ is larger at the upper levels, and even more pronounced at the cloud edge because DSD broadening is strong due to collisioncoalescence (clean; Fig. 11d). The resulting large drops contribute more to the increase in $\sigma$ than to the increase in the mean radius. Another proxy for the width of the DSD is the ratio of the effective radius to the mean volume radius, which is around 1.04-1.14 for the polluted cloud (Fig. 12b), in agreement with observations (Martin et al. 1994; Freud and Rosenfeld 2012). This ratio exceeds 1.1 in zones of 

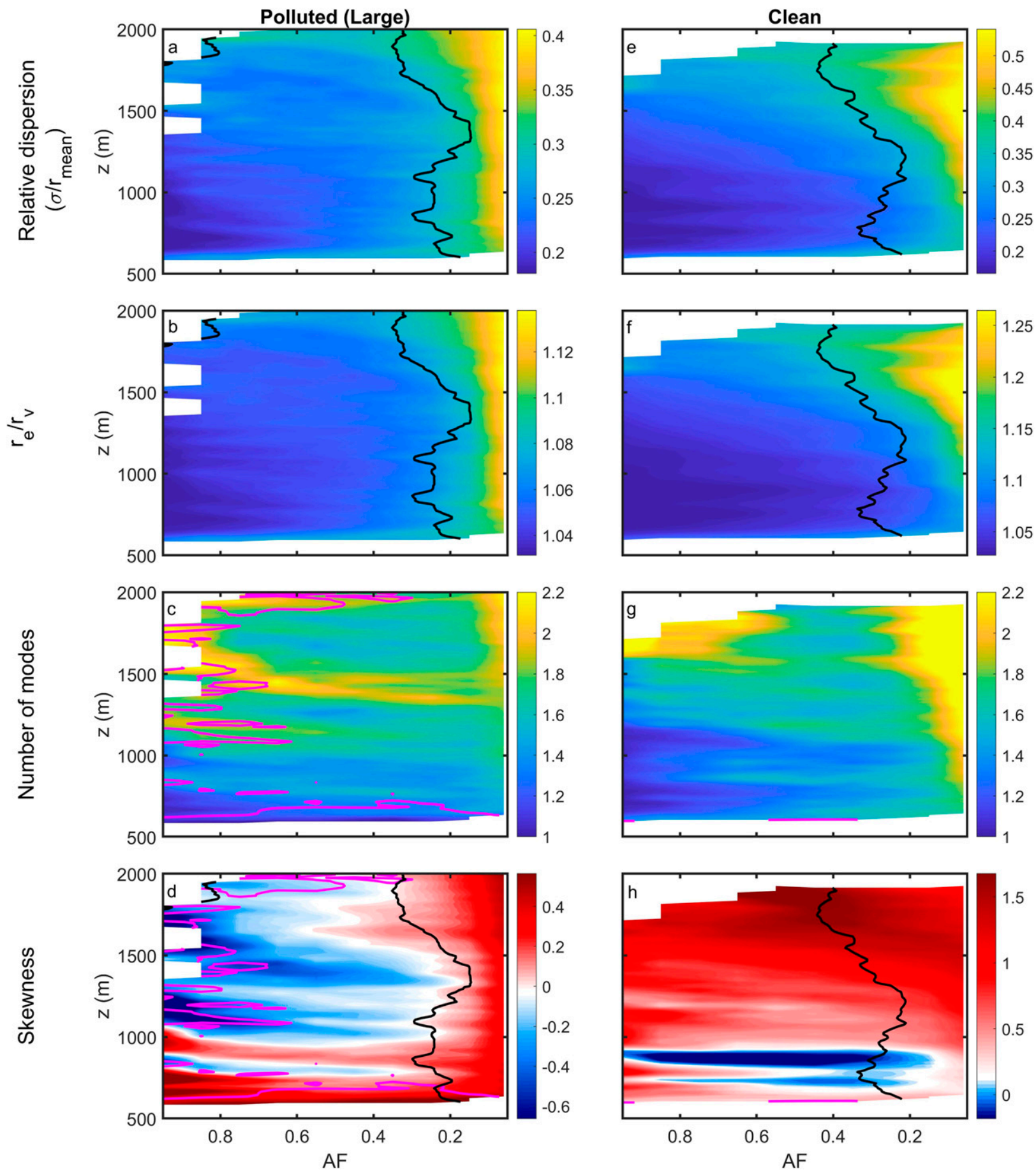

FIG. 12. DSD properties in the $z-\mathrm{AF}$ space for (left) large polluted and (right) clean $\mathrm{Cu}$. (top) DSD relative dispersion. (second row) The ratio of effective radius to mean volume radius. (third row) Number of modes. (bottom) DSD skewness. Notations are as follows: Magenta contours mark the region of secondary nucleation $\left(0.4 \mathrm{~cm}^{-3} \mathrm{~s}^{-1}\right)$. The black contour denotes zero supersaturation, so evaporation occurs on its right side. Note that the number of modes of individual DSDs is an integer, but the $z$-AF space represents mean values that appear with decimal figures. The color scales in (d) and (h) are different.

collision-coalescence and cloud edges, since the value of the effective radius is more sensitive to the fraction of the largest droplets.
The third row shows the number of DSD modes. As mentioned above, there are three main mechanisms for the formation of multimodal DSDs: 1) mixing of cloud volumes with 
different DSDs (this process is especially effective near cloud edges), 2) in-cloud nucleation due to an increase in supersaturation, and 3) collision-coalescence.

We calculated the number of modes in the DSDs by counting the number of peaks for each individual DSD. Some parameters were determined empirically and used in the algorithm to avoid counting of adjacent peaks. The accuracy of the algorithm was tested by visually examining 845 randomly chosen DSDs and estimating their number of modes. Complete agreement for the number of modes was found for $70 \%$ of the cases while an agreement of $85 \%$ was achieved for the binary classification of multimodality vs. monomodality. We emphasize that the multimodality is not restricted to the separation of the DSD to cloud droplets and raindrops/drizzle. Most modes were within the size range of cloud droplets $(r<40 \mu \mathrm{m})$, as shown in Fig. 9. Figures $12 \mathrm{c}$ and $12 \mathrm{~g}$ show that in polluted cloud, multimodal (bimodal) DSDs arise mostly in the cloud core via in-cloud nucleation. In clean clouds, multimodal DSDs arise mainly in the cloud core near the cloud top, where collision-coalescence is intense. Both clouds contain multimodal DSDs in the vicinity to cloud edge, due to mixing of very different DSDs. As shown below (Fig. 12), a secondary peak due to in-cloud nucleation in the cloud core does not exist in the clean cloud scenario because all available CCNs were activated at cloud base. During the growth and mature stages of the large polluted cloud, $20 \%-50 \%$ of the grid points contained multimodal DSD. This is in agreement with observational reports (Schmeissner et al. 2015; Lasher-Trapp et al. 2005; Yeom et al. 2019).

The mechanisms of the DSD formation are manifested in the $z$-AF space by the value of DSD skewness, defined as the ratio of the third central moment and the cube of the standard deviation. Liu et al. (2017) reported a wide range of skewness values from -2 to 5 , with a mean value of 0.2 and largest values at the edge of the cloud. The skewness map in the $z-\mathrm{AF}$ space shows abrupt changes in sign (Figs. 12d,g), which shows that the skewness is strongly related to the microphysical processes. The skewness near cloud base at the core of the polluted cloud is positive (Fig. 12d). This is a direct consequence of the process of droplet activation and $\mathrm{CCN}$ size distribution. Most CCN in the aerosol distribution are small while the number of large $\mathrm{CCN}$ is very low; correspondingly, the concentration of the droplets decreases with droplet size. At the cloud core, the skewness becomes negative as a result of diffusion growth and in-cloud nucleation.

This negative skewness turns to positive in the transition zone near the cloud edge, where evaporation dominates. During evaporation the radius of smaller droplets decreases faster, leading to DSDs with many small droplets and a few large ones, resulting in a positive skewness. Figure 9 shows that the DSDs in the transition zone $(\mathrm{AF}<0.2)$ are composed of many small droplets and a few regular-sized ones that are in the size range of the droplets from the adiabatic regions. This suggests that evaporation plays an important role in the broadening of the DSD. Detailed analysis of the DSD shape change during evaporation is presented in Pinsky et al. (2016) (see Fig. 7 in their paper), where the formation of positive skewness is obvious. Note that dilution alone decreases droplet concentration, but does not affect the DSD shape. Figure $12 \mathrm{~h}$ shows that the DSD skewness of the clean cloud differs substantially from that of polluted clouds. The skewness is mostly positive and does not exhibit dependence on AF, especially at high levels. This is a result of collision-coalescence in the clean cloud leading to increase in the concentration of large drops. This makes skewness insensitive to AF, but sensitive to the distance from cloud base. The weight of the largest droplets is very large near cloud top, resulting in a skewness $>1$. On the other hand, there is no in-cloud nucleation, so there are no counteracting processes that decrease skewness.

We therefore observe that the shape of the DSDs and the main DSD parameters in nonprecipitating $\mathrm{Cu}$ can be classified according to AF. Regarding the DSD skewness in clean clouds at their maximum development stage, it is determined by the collision-coalescence, and increases with height.

\section{Summary and conclusions}

The microphysical properties in nonprecipitating cumulus clouds were investigated in the prism of adiabatic processes versus dilution and mixing. A LES model coupled with a spectral bin microphysics scheme was used to simulate isolated single clouds at high resolution of $10 \mathrm{~m}$ in different sizes and aerosols concentration.

The adiabatic fraction (AF) was used as a measure of adiabaticity, to study mixing effects on several cloud macro- and microphysical properties (e.g., updraft, microphysical processes rates, and droplet size distribution shapes). It is shown that using AF can simplify the investigation of mixing effects on droplet size distribution (DSD) by enabling to separate mixing into two processes: 1) mechanical mixing, which acts to dilute cloudy volumes and superimpose their properties (as the mixing of a passive scalar), and 2) mixing with droplets evaporation (which is often modeled as homogeneous or inhomogeneous). We show that in cloud zones with $\mathrm{AF}>0.2$, the relative humidity $(\mathrm{RH}) \sim 100 \%$ and the AF should behave as a passive scalar as the mixing is mechanical (at such high RH homogeneous and inhomogeneous mixing types are indistinguishable). At cloud edges where $\mathrm{AF}<0.2$ and $\mathrm{RH}<$ $100 \%$, AF changes by evaporation and affects differently the DSDs. The comparison of fields of AF and those of passive scalars was shown to provide important information about the nature of mixing, by Eytan et al. (2021).

The properties of AF allow analyzing the continues trends of such variables with respect to mixing, and to separate the cloud to three zones with different properties that are determined by different processes: core, transition zone (near cloud edge), and intermediate zone. While the adiabatic core is simpler to consider and can be thought of as the skeleton of the cloud and the source for cloudy volumes, the transition zone that acts as a sink, also adds complexity due its turbulent and inhomogeneous nature. This line of thought is in accordance with the work of Pinsky et al. (2021a,b) that used wavelet filters to separate the flow in the cloud to convective and turbulent motions, and show its relations to moist adiabatic processes. 
Since in situ observations record the data as distance along the instrument's trajectory, we investigated the changes of cloud properties as a function of the distance to the nearest cloud edge (DFCE). Our simulations results (using DFCE) are supported by high-frequency measurements, and show cloud structure features, such as the subsiding shell and interface zone at a width of $20-40 \mathrm{~m}$ that is separating the cloud body from the surrounding air (Gerber et al. 2008; Katzwinkel et al. 2014; Schmeissner et al. 2015). Such transition zones, marked by a sharp decrease in $q_{c}$ and RH (Fig. 2) are related to volumes with $\mathrm{AF}<0.2$. $\mathrm{AF}$ is shown to increase with DFCE, but the relations and their robustness seem to change with cloud life cycle and altitude (higher levels contain "older" parcels/parts of the cloud). The classification of cloud microphysical properties according to AF was shown to provide a more stable representation of the cloud structure compared to DFCE.

\section{a. Occurrence and location of adiabatic volumes}

Large- and medium-sized $\mathrm{Cu}$ contain an adiabatic core (AF > 0.9 ), whose size decreases with height, constituting $10 \%-50 \%$ of the cloud area below the inversion. The adiabatic core's area fraction decreases with height until it breaks into small fragments above the inversion. Nevertheless, local high AF values were found in almost all levels, for all cloud sizes during the growth stage (first row in Fig. 7). The buoyancy, generated by latent heat release in the core volumes determines the cloud-top height. Moreover, small adiabatic parcels that reach the highest level are sources of large droplets that can initiate collision-coalescence and raindrop formation. The occurrence of adiabatic regions in shallow $\mathrm{Cu}$ is much debated and barely reported in in situ measurement studies. The doubts regarding the existence of undiluted (or slightly diluted) zones might be a consequence of the limitation of aircraft measurements to sample the cloud in the right time and space. Indeed, the results show that such zones near cloud top exist for a short duration (about $1 \mathrm{~min}$ ) with a small area fraction $(<0.1)$, leading to a probability of $\sim 1 \%$ to measure these volumes. Moreover, the small fraction of the adiabatic core at high altitudes might be missed when sampled in coarse resolutions. Note that most in situ measurements are acquired near the cloud tops (for both airplanes and helicopters; Gerber et al. 2008; Katzwinkel et al. 2014; Schmeissner et al. 2015). Nevertheless, it is remarkable that the cloud volumes with $\mathrm{AF}>0.7-0.8$ were registered in high-resolution measurements (Brenguier et al. 2013, p. 304; Gerber 2000). The small cloud did not contain an adiabatic core; such clouds are often forced by initial fluctuations or by thermal instability of the cloud atmospheric layer.

\section{b. Relations of the microphysics to $A F$}

The comparison with available high-frequency in situ measurements showed high similarity of DSD shapes and parameters, like DSD width, dispersion, effective radius, ratio of effective radius to the mean volume radius, and skewness. We found that the shapes of DSD and their parameters could be properly classified (separated) according to AF values (i.e.,
DSDs' shapes are determined by the adiabaticity level that is related to different microphysical processes). The strong relations between the shape and width of the DSD to AF and its related processes suggest that artificial numerical broadening in SBM schemes should be negligible compared to natural physical processes. Those processes are best represented by the SBM schemes.

DSDs in the adiabatic cores (with AF $>0.9$ ) are highly similar, with a low relative dispersion slightly dependent on height in the first few hundreds of meters above cloud base. Above these levels, in the case of a polluted cloud, secondary nucleation may take place in the adiabatic core, causing the formation of a small second mode of smallest droplets, which increase the relative dispersion. At the mature stage of the clean cloud (which activates all of its droplets near cloud base), relative dispersion increase with height only at the level where collision-coalescence is initiated. The skewness of DSDs (characterizing the DSD shape) was also found to agree with in situ measurements (e.g., Liu et al. 2017). In the polluted cloud, the skewness was found to depend on AF, and changes its sign due to different microphysical processes that occur in the cloud core or periphery. In the cloud core, diffusion growth, secondary nucleation and the lack of large droplets form a DSD shape with negative skewness. In the clean cloud, collision-coalescence at the mature stage leads to the appearance of large drops tail that mask the role of AF in DSD skewness.

The DSDs in the transition zone, where $\mathrm{AF}<0.2$ are multimodal, with a relative dispersion much larger than in other cloud regions. These DSDs that come in many different shapes (Figs. 9 and 10) mostly contain only small droplets, although some may also contain large droplets (depending on the history of the cloud air parcels). Because the radii of smaller droplets decrease faster during evaporation, the skewness in the transition zone is highly positive. This shows that $\mathrm{AF}$ and skewness contain important information about dominating microphysical processes. It was found that during the growing and mature stages of the large polluted cloud, $20 \%-50 \%$ of the grid points contained multimodal DSD. This agrees with observational reports (Schmeissner et al. 2015; Lasher-Trapp et al. 2005; Yeom et al. 2019). However, the reasons of the formation of the multimodal DSDs are different in the cores and at cloud periphery. In the cloud core the multimodal DSDs form due to in-cloud activation of small aerosol particles that were not activated at cloud base. At cloud periphery the multimodal DSDs form due to mixing of volumes with different DSDs.

The effective radius $\left(r_{e}\right)$ was found to vary only slightly in the horizontal direction for the polluted clouds, in agreement with results reported in observations. (Freud et al. 2008; Pawlowska et al. 2006; Zhang et al. 2011; Prabha et al. 2011; Konwar et al. 2021); $r_{e}$ is experiencing changes only in the narrow transition zone (i.e., when evaporation is significant). It shows that in clouds that contain an adiabatic core, the vertical profile of $r_{e}$ in the core can well represent the profile of the entire cloud (since it is nearly constant in the horizontal direction). Moreover, the low variability of the DSD shapes in zones of high and intermediate AF should simplify and improve the parameterization of the DSD formation in microphysical schemes. 


\section{c. Future directions}

This study examines the simplest case in which aerosols enter the cloud from its base. The role of entraining aerosols above the cloud base should be studied in more detail in future research, together with the effects of aerosol release by complete droplet evaporation. Recent works reported that a substantial fraction of droplets originates by activation of lateral entrained aerosols. Hoffmann et al. (2015) dealt with trade cumulus clouds and found that the relative contribution of lateral entrained aerosols to the total number concentration is less than $15 \%$ during the growing stage, and can reach $60 \%$ in the dissipating stage (see Fig. 10 in their paper). Chandrakar et al. (2021) simulated deeper Congestus clouds under a constant aerosol-mixing ratio throughout the entire atmospheric column and found that $20 \%$ of the droplets originated from lateral entrainment of aerosols. The distribution of aerosols at high altitudes gives a higher bound or might overestimate the effect since aerosol concentration typically decreases with height above the surface. We argue that the similarity of our results to high-frequency measurements shows that the DSDs obtained using this simplified assumption are realistic.

Considering the occurrence and potential importance of smallscale regions, such as the transition zone of the cloud's edge, and small adiabatic volumes at high levels emphasizes the need of LES studies with high resolution. These simulations should include detailed representation of the microphysics in order to study the effects of mixing and other microphysical processes on the shape of the droplet size distribution in those regions.

We conclude that the utilization of AF as a measure of adiabaticity for characterizing cloud structure turned out to be quite fruitful. At the same time, some important questions concerning the role of entrainment and mixing require further investigation. While the mechanisms leading to rapid formation of the cloud structure at the core and the edge of the cloud (transition zone) are clear, the mechanisms of the formation of the wide zone of the intermediate AF values have not been clarified yet. The questions that arise include these: Is it only diluted core volumes, or does it experience bidirectional effects from the edge? Does it contain other unique processes? All of these questions will be dependent on the mixing process, which could be either due to turbulent or coherent motions, like the toroidal vortex.

Acknowledgments. This project has received funding from the European Research Council (ERC), under the European Union's Horizon 2020 research and innovation programme (CloudCT, Grant Agreement 810370). A. Khain and M. Pinsky were supported by the grants from the Department of Energy (DE964SC0008811) and by the Israel Science Foundation (Grants 2027/ 17 and 2635/20). E. Eytan was supported by the Weizmann Institute Sustainability and Energy Research Initiative.

Data availability statement. The microphysical and thermodynamical profiles used to initialize the simulation can be obtained upon request from the corresponding author. The SAM codes should be requested from Prof. Marat Khairoutdinov from the School of Marine and Atmospheric Sciences, Stony Brook University.

\section{APPENDIX}

\section{Adiabatic Fraction Calculations}

There are a few approaches for obtaining $\mathrm{LWC}_{\mathrm{ad}}$, which are discussed and tested in a different work (Eytan et al. 2021). In this work, we use the analytical derivation of Korolev and Mazin (2003), which was found to be the most accurate.

The equation for supersaturation $(S)$ in an adiabatically ascending parcel is

$$
\frac{d \log (S+1)}{d z}=A_{1}-A_{2} \frac{d \mathrm{LWC}}{d z}
$$

where $\quad A_{1}=(g / T)\left[\left(L_{w} / c_{p} R_{v} T\right)-\left(1 / R_{a}\right)\right] ; \quad A_{2}=\left(1 / \rho_{v}\right)$ $+\left(L_{w}^{2} / c_{p} R_{v} T^{2} \rho_{a}\right)$, and $S$ is supersaturation, $g$ is the gravity acceleration, $T$ is temperature, $L_{w}$ is the latent heat of evaporation, $c_{p}$ is the heat capacity of air under constant pressure, $R_{v}$ and $R_{a}$ are the gas constants of water vapor and dry air, respectably, and $\rho_{v}$ and $\rho_{a}$ are the density of water vapor and dry air, respectably.

The first term on the RHS is a source of $S$ resulting from cooling of the ascending parcel. The second term is a sink due to latent heat release and water vapor loss by condensation.

LWC can be considered as $\mathrm{LWC}_{\mathrm{ad}}$ because Eq. (2) describes an adiabatic parcel model.

Since typically $S \ll 1$, we can use $\log (S+1) \approx S$ and obtain $\mathrm{LWC}_{\mathrm{ad}}$ as

$$
\mathrm{LWC}_{\mathrm{ad}(z)} \approx \int_{0}^{z} \frac{A_{1\left(z^{\prime}\right)}}{A_{2\left(z^{\prime}\right)}} d z^{\prime}-\int_{0}^{z} \frac{1}{A_{2\left(z^{\prime}\right)}} \frac{d S}{d z} d z^{\prime}
$$

where $z=0$ at cloud base. One can see that $\mathrm{LWC}_{\mathrm{ad}}$ is not universal and is not only a function of $z$, but also depends on temperature, humidity (via parameters $A_{1}$ and $A_{2}$ ), vertical velocity, and aerosols, which affect supersaturation. We note that AF calculations usually use the saturation adjustments assumption, considering $S \approx 0$ everywhere in the cloud, and dismissing the second term in Eq. (3). This assumption underestimates AF in regions of high $S$ (e.g., near cloud base, and in clouds with low droplets concentration). Here, the high-resolution, spectral bin microphysics (SBM) model allows considering the complete equation of $\mathrm{LWC}_{\mathrm{ad}}$ and comparing cases with different aerosols concentrations.

The solution of the Lagrangian parcel model is extracted from the Eulerian model outputs by assuming stationarity of the thermodynamic profiles for the few minutes in which a parcel ascends during the growth and mature stage of the cloud (i.e., assuming the profiles of temperature and humidity can be used to predict the conditions a parcel in the cloud base will be exposed to). The profiles are taken from the most adiabatic regions in the cloud (where humidity is maximal and temperature is warmer due to latent heating) by averaging the points with the highest $-5 \%$ updraft values at each level. 


\section{REFERENCES}

Abma, D., T. Heus, and J. P. Mellado, 2013: Direct numerical simulation of evaporative cooling at the lateral boundary of shallow cumulus clouds. J. Atmos. Sci., 70, 2088-2102, https:// doi.org/10.1175/JAS-D-12-0230.1.

Altaratz, O., I. Koren, T. Reisin, A. Kostinski, G. Feingold, Z. Levin, and Y. Yin, 2008: Aerosols' influence on the interplay between condensation, evaporation and rain in warm cumulus cloud. Atmos. Chem. Phys., 8, 15-24, https://doi.org/10. 5194/acp-8-15-2008.

Baker, M. B., R. G. Corbin, and J. Latham, 1980: The influence of entrainment on the evolution of cloud droplet spectra: I. A model of inhomogeneous mixing. Quart. J. Roy. Meteor. Soc., 106, 581-598, https://doi.org/10.1002/qj.49710644914.

Beard, K. V., 1976: Terminal velocity and shape of cloud and precipitation drops aloft. J. Atmos. Sci., 33, 851-864, https://doi. org/10.1175/1520-0469(1976)033<0851:TVASOC >2.0.CO;2.

Benmoshe, N., A. P. Khain, M. Pinsky, and A. Pokrovsky, 2012: Turbulent effects on cloud microstructure and precipitation of deep convective clouds as seen from simulations with a 2D spectral microphysics cloud model. J. Geophys. Res., 117, D06220, https://doi.org/10.1029/2011JD016603.

Bera, S., 2021: Droplet spectral dispersion by lateral mixing process in continental deep cumulus clouds. J. Atmos. Sol.-Terr. Phys., 214, 105550, https://doi.org/10.1016/j.jastp.2021.105550.

Bony, S., and Coauthors, 2015: Clouds, circulation and climate sensitivity. Nat. Geosci., 8, 261-268, https://doi.org/10.1038/ ngeo2398.

Bott, A., 1998: A flux method for the numerical solution of the stochastic collection equation. J. Atmos. Sci., 55, 2284-2293, https://doi.org/10.1175/1520-0469(1998)055<2284:AFMFTN $>2$. $0 . \mathrm{CO} ; 2$.

Brenguier, J.-L., and Coauthors, 2013: In situ measurements of cloud and precipitation particles. Airborne Measurements for Environmental Research: Methods and Instruments, M. Wendisch and J.-L. Brenguier, Eds., Wiley, 225-301.

Cahalan, R. F., and J. H. Joseph, 1989: Fractal statistics of cloud fields. Mon. Wea. Rev., 117, 261-272, https://doi.org/10.1175/ 1520-0493(1989)117<0261:FSOCF > 2.0.CO;2.

Ceppi, P., F. Brient, M. D. Zelinka, and D. L. Hartmann, 2017: Cloud feedback mechanisms and their representation in global climate models. Wiley Interdiscip. Rev.: Climate Change, 8, e465, https://doi.org/10.1002/wcc.465.

Chandrakar, K. K., W. W. Grabowski, H. Morrison, and G. H. Bryan, 2021: Impact of entrainment-mixing and turbulent fluctuations on droplet size distributions in a cumulus cloud: An investigation using Lagrangian microphysics with a subgrid-scale model. J. Atmos. Sci., 78, 2983-3005, https://doi.org/ 10.1175/JAS-D-20-0281.1.

Dawe, J. T., and P. H. Austin, 2012: Statistical analysis of an LES shallow cumulus cloud ensemble using a cloud tracking algorithm. Atmos. Chem. Phys., 12, 1101-1119, https://doi.org/10. 5194/acp-12-1101-2012.

Eytan, E., I. Koren, O. Altaratz, M. Pinsky, and A. Khain, 2021: Revisiting adiabatic fraction in cumulus clouds: High-resolution simulations with passive tracer. Atmos. Chem. Phys., 21, 16203-16217, https://doi.org/10.5194/acp-21-16203-2021.

Fan, J., M. Ovtchinnikov, J. M. Comstock, S. A. McFarlane, and A. Khain, 2009: Ice formation in Arctic mixed-phase clouds: Insights from a 3-D cloud-resolving model with size-resolved aerosol and cloud microphysics. J. Geophys. Res., 114, D04205, https://doi.org/10.1029/2008JD010782.
Freud, E., and D. Rosenfeld, 2012: Linear relation between convective cloud drop number concentration and depth for rain initiation. J. Geophys. Res., 117, D02207, https://doi.org/10. 1029/2011JD016457.

,-- M. O. Andreae, A. A. Costa, and P. Artaxo, 2008: Robust relations between $\mathrm{CCN}$ and the vertical evolution of cloud drop size distribution in deep convective clouds. Atmos. Chem. Phys., 8, 1661-1675, https://doi.org/10.5194/acp-8-16612008.

Gerber, H., 1996: Microphysics of marine stratocumulus clouds with two drizzle modes. J. Atmos. Sci., 53, 1649-1662, https:// doi.org/10.1175/1520-0469(1996)053<1649:MOMSCW>2.0. $\mathrm{CO} ; 2$.

, 2000: Structure of small cumulus clouds. Proc. 13th Int. Conf. on Clouds and Precipitation, Reno, NV, International Commission on Clouds and Precipitation of the International Association of Meteorology and Atmospheric Sciences, 105-108.

—, G. M. Frick, J. B. Jensen, and J. G. Hudson, 2008: Entrainment, mixing, and microphysics in trade-wind cumulus. $J$. Meteor. Soc. Japan, 86A, 87-106, https://doi.org/10.2151/jmsj. 86A.87.

Heiblum, R. H., and Coauthors, 2016a: Characterization of cumulus cloud fields using trajectories in the center of gravity versus water mass phase space: 1 . Cloud tracking and phase space description. J. Geophys. Res. Atmos., 121, 6336-6355, https://doi.org/10.1002/2015JD024186.

— - and Coauthors, 2016b: Characterization of cumulus cloud fields using trajectories in the center of gravity versus water mass phase space: 2. Aerosol effects on warm convective clouds. J. Geophys. Res. Atmos., 121, 6356-6373, https://doi. org/10.1002/2015JD024193.

Heus, T., and H. J. J. Jonker, 2008: Subsiding shells around shallow cumulus clouds. J. Atmos. Sci., 65, 1003-1018, https://doi. org/10.1175/2007JAS2322.1.

— , G. van Dijk, H. J. J. Jonker, and H. E. A. Van den Akker, 2008: Mixing in shallow cumulus clouds studied by Lagrangian particle tracking. J. Atmos. Sci., 65, 2581-2597, https://doi. org/10.1175/2008JAS2572.1.

- , H. J. J. Jonker, H. E. A. Van den Akker, E. J. Griffith, M. Koutek, and F. H. Post, 2009: A statistical approach to the life cycle analysis of cumulus clouds selected in a virtual reality environment. J. Geophys. Res., 114, D06208, https://doi. org/10.1029/2008JD010917.

Hoffmann, F., H. Siebert, J. Schumacher, T. Riechelmann, J. Katzwinkel, B. Kumar, P. Götzfried, and S. Raasch, 2014: Entrainment and mixing at the interface of shallow cumulus clouds: Results from a combination of observations and simulations. Meteor. Z., 23, 349-368, https://doi.org/10.1127/09412948/2014/0597.

— S. Raasch, and Y. Noh, 2015: Entrainment of aerosols and their activation in a shallow cumulus cloud studied with a coupled LCM-LES approach. Atmos. Res., 156, 43-57, https://doi.org/10.1016/j.atmosres.2014.12.008.

Houze, R. A., 2014: Basic cumulus dynamics. Cloud Dynamics. International Geophysics Series, Vol. 104, Elsevier, 165-185.

Ilotoviz, E., and A. Khain, 2016: Application of a new scheme of cloud base droplet nucleation in a spectral (bin) microphysics cloud model: Sensitivity to aerosol size distribution. Atmos. Chem. Phys., 16, 14317-14329, https://doi.org/10.5194/acp-1614317-2016.

IPCC, 2013: Climate Change 2013: The Physical Science Basis. Cambridge University Press, 1535 pp., https://doi.org/10.1017/ CBO9781107415324. 
Jaenicke, R., 1987: Physical and Chemical Properties of the Air, G. Fischer, Ed., Springer, 570 pp.

Katzwinkel, J., H. Siebert, T. Heus, and R. A. Shaw, 2014: Measurements of turbulent mixing and subsiding shells in trade wind cumuli. J. Atmos. Sci., 71, 2810-2822, https://doi.org/10. 1175/JAS-D-13-0222.1.

Khain, A. P., and M. Pinsky, 2018: Physical Processes in Clouds and Cloud Modeling. Cambridge University Press, 626 pp.

- M. Ovtchinnikov, M. Pinsky, A. Pokrovsky, and H. Krugliak, 2000: Notes on the state-of-the-art numerical modeling of cloud microphysics. Atmos. Res., 55, 159-224, https://doi.org/ 10.1016/S0169-8095(00)00064-8.

- A. Pokrovsky, M. Pinsky, A. Seifert, and V. Phillips, 2004: Simulation of effects of atmospheric aerosols on deep turbulent convective clouds using a spectral microphysics mixedphase cumulus cloud model. Part I: Model description and possible applications. J. Atmos. Sci., 61, 2963-2982, https:// doi.org/10.1175/JAS-3350.1.

— N. Nenmoshe, and A. Pokrovsky, 2008: Factors determining the impact of aerosols on surface precipitation from clouds: An attempt at classification. J. Atmos. Sci., 65, 1721-1748, https://doi.org/10.1175/2007JAS2515.1.

- , T. V. Prabha, N. Benmoshe, G. Pandithurai, and M. Ovchinnikov, 2013: The mechanism of first raindrops formation in deep convective clouds. J. Geophys. Res. Atmos., 118, 9123-9140, https://doi.org/10.1002/jgrd.50641.

Khain, P., and Coauthors, 2019: Parameterization of vertical profiles of governing microphysical parameters of shallow cumulus cloud ensembles using LES with bin microphysics. $J$. Atmos. Sci., 76, 533-560, https://doi.org/10.1175/JAS-D-180046.1 .

Khairoutdinov, M. F., and Y. L. Kogan, 1999: A large eddy simulation model with explicit microphysics: Validation against aircraft observations of a stratocumulus-topped boundary layer. J. Atmos. Sci., 56, 2115-2131, https://doi.org/10.1175/ 1520-0469(1999)056<2115:ALESMW > 2.0.CO;2.

, and D. A. Randall, 2003: Cloud resolving modeling of the ARM summer 1997 IOP: Model formulation, results, uncertainties, and sensitivities. J. Atmos. Sci., 60, 607-625, https://doi. org/10.1175/1520-0469(2003)060<0607:CRMOTA >2.0.CO;2.

Kim, B.-G., M. A. Miller, S. E. Schwartz, Y. Liu, and Q. Min, 2008: The role of adiabaticity in the aerosol first indirect effect. J. Geophys. Res., 113, D05210, https://doi.org/10.1029/ 2007JD008961.

Kogan, Y. L., 1991: The simulation of a convective cloud in a 3-D model with explicit microphysics. Part I: Model description and sensitivity experiments. J. Atmos. Sci., 48, 1160-1189, https:// doi.org/10.1175/1520-0469(1991)048<1160:TSOACC > 2.0.CO;2.

Konwar, M., T. Prabhakaran, A. Khain, and M. Pinsky, 2021: Cloud microphysical structure analysis based on high-resolution in situ measurements. J. Atmos. Sci., 78, 2265-2285, https://doi.org/10.1175/JAS-D-20-0229.1.

Koren, I., L. Oreopoulos, G. Feingold, L. A. Remer, and O. Altaratz, 2008: How small is a small cloud?Atmos. Chem. Phys., 8, 3855-3864, https://doi.org/10.5194/acp-8-3855-2008.

Korolev, A. V., 1994: A study of bimodal droplet size distributions in stratiform clouds. Atmos. Res., 32, 143-170, https:// doi.org/10.1016/0169-8095(94)90057-4.

— , and I. P. Mazin, 2003: Supersaturation of water vapor in clouds. J. Atmos. Sci., 60, 2957-2974, https://doi.org/10.1175/ 1520-0469(2003)060<2957:SOWVIC $>2.0$. CO;2.

Kumar, B., S. Bera, T. V. Prabha, and W. W. Grabowski, 2017: Cloud-edge mixing: Direct numerical simulation and observations in Indian monsoon clouds. J. Adv. Model. Earth Syst., 9, 332-353, https://doi.org/10.1002/2016MS000731.

Lasher-Trapp, S. G., W. A. Cooper, and A. M. Blyth, 2005: Broadening of droplet size distributions from entrainment and mixing in a cumulus cloud. Quart. J. Roy. Meteor. Soc., 131, 195-220, https://doi.org/10.1256/qj.03.199.

Levin, Z., E. Ganor, and V. Gladstein, 1996: The effects of desert particles coated with sulfate on rain formation in the eastern Mediterranean. J. Appl. Meteor., 35, 1511-1523, https://oi. org/10.1175/1520-0450(1996)035<1511:TEODPC>2.0.CO;2.

Liu, Y., P. H. Daum, and S. S. Yum, 2006: Analytical expression for the relative dispersion of the cloud droplet size distribution. Geophys. Res. Lett., 33, L02810, https://doi.org/10.1029/ 2005 GL024052.

, C. Lu, and W. Li, 2017: Skewness of cloud droplet spectrum and an improved estimation for its relative dispersion. Meteor. Atmos. Phys., 129, 47-56, https://doi.org/10.1007/ s00703-016-0458-9.

Magaritz-Ronen, L., M. Pinsky, and A. Khain, 2014: Effects of turbulent mixing on the structure and macroscopic properties of stratocumulus clouds demonstrated by a Lagrangian trajectory model. J. Atmos. Sci., 71, 1843-1862, https://doi.org/10. 1175/JAS-D-12-0339.1.

Martin, G. M., D. W. Johnson, and A. Spice, 1994: The measurement and parameterization of effective radius of droplets in warm stratocumulus clouds. J. Atmos. Sci., 51, 1823-1842, https://doi.org/10.1175/1520-0469(1994)051<1823:TMAPOE $>2$. $0 . \mathrm{CO} ; 2$.

Mellado, J. P., C. S. Bretherton, B. Stevens, and M. C. Wyant, 2018: DNS and LES for simulating stratocumulus: Better together. J. Adv. Model. Earth Syst., 10, 1421-1438, https:// doi.org/10.1029/2018MS001312.

Morrison, H., M. Witte, G. H. Bryan, J. Y. Harrington, and Z. J. Lebo, 2018: Broadening of modeled cloud droplet spectra using bin microphysics in an Eulerian spatial domain. $J$. Atmos. Sci., 75, 4005-4030, https://doi.org/10.1175/JAS-D-180055.1.

Neggers, R. A. J., H. J. J. Jonker, and A. P. Siebesma, 2003: Size statistics of cumulus cloud populations in large-eddy simulations. J. Atmos. Sci., 60, 1060-1074, https://doi.org/10.1175/ 1520-0469(2003)60<1060:SSOCCP>2.0.CO;2.

Nuijens, L., and A. P. Siebesma, 2019: Boundary layer clouds and convection over subtropical oceans in our current and in a warmer climate. Curr. Climate Change Rep., 5, 80-94, https:// doi.org/10.1007/s40641-019-00126-x.

Ovtchinnikov, M., and Y. L. Kogan, 2000: An investigation of ice production mechanisms in small cumuliform clouds using a 3D model with explicit microphysics. Part I: Model description. J. Atmos. Sci., 57, 2989-3003, https://doi.org/10.1175/ 1520-0469(2000)057<2989:AIOIPM>2.0.CO;2.

Pandithurai, G., S. Dipu, T. V. Prabha, R. S. Maheskumar, J. R. Kulkarni, and B. N. Goswami, 2012: Aerosol effect on droplet spectral dispersion in warm continental cumuli. J. Geophys. Res. 117, D16202, https://doi.org/10.1029/2011JD016532.

Pardo, L. H., H. Morrison, L. A. T. Machado, J. Y. Harrington, and Z. J. Lebo, 2020: Drop size distribution broadening mechanisms in a bin microphysics Eulerian model. J. Atmos. Sci., 77, 3249-3273, https://doi.org/10.1175/JAS-D-20-0099.1.

Pawlowska, H., W. W. Grabowski, and J. L. Brenguier, 2006: Observations of the width of cloud droplet spectra in stratocumulus. Geophys. Res. Lett., 33, L19810, https://doi.org/10. 1029/2006GL026841. 
Pinsky, M., and A. Khain, 2002: Effects of in-cloud nucleation and turbulence on droplet spectrum formation in cumulus clouds. Quart. J. Roy. Meteor. Soc., 128, 501-533, https://doi.org/10. 1256/003590002321042072.

- _ - and M. Shapiro, 2001: Collision efficiency of drops in a wide range of Reynolds numbers: Effects of pressure on spectrum evolution. J. Atmos. Sci., 58, 742-764, https://doi. org/10.1175/1520-0469(2001)058<0742:CEODIA $>2.0$. CO;2.

,$-\frac{}{-}$, A. Korolev, and L. Magaritz-Ronen, 2016: Theoretical investigation of mixing in warm clouds-Part 2: Homogeneous mixing. Atmos. Chem. Phys., 16, 9255-9272, https://doi. org/10.5194/acp-16-9255-2016.

— - E. Eytan, I. Koren, O. Altaratz, and A. Khain, 2021a: Convective and turbulent motions in non-precipitating $\mathrm{Cu}$. Part 1: Method of separation of convective and turbulent motions. $J$. Atmos. Sci., 78, 2307-2321, https://doi.org/10.1175/JAS-D-200127.1.

,,--- , and A. Khain, 2021b: Convective and turbulent motions in non-precipitating $\mathrm{Cu}$. Part 2: LES simulated cloud represented by a starting plume. J. Atmos. Sci., https://doi. org/10.1175/JAS-D-21-0137.1, in press.

Prabha, T. V., A. Khain, R. S. Maheshkumar, G. Pandithurai, J. R. Kulkarni, M. Konwar, and B. N. Goswami, 2011: Microphysics of premonsoon and monsoon clouds as seen from in situ measurements during the Cloud Aerosol Interaction and Precipitation Enhancement Experiment (CAIPEEX). $J$. Atmos. Sci., 68, 1882-1901, https://doi.org/10.1175/ 2011JAS3707.1.

Rodts, S. M. A., P. G. Duynkerke, and H. J. J. Jonker, 2003: Size distributions and dynamical properties of shallow cumulus clouds from aircraft observations and satellite data. J. Atmos. Sci., 60, 1895-1912, https://doi.org/10.1175/1520-0469(2003)060<1895: SDADPO $>2.0 . \mathrm{CO} ; 2$.

Romps, D. M., and Z. Kuang, 2010a: Do undiluted convective plumes exist in the upper tropical troposphere? J. Atmos. Sci., 67, 468-484, https://doi.org/10.1175/2009JAS3184.1.

- , and,$- 2010 \mathrm{~b}$ : Nature versus nurture in shallow convection. J. Atmos. Sci., 67, 1655-1666, https://doi.org/10.1175/ 2009JAS3307.1.

Rosenfeld, D., and G. Gutman, 1994: Retrieving microphysical properties near the tops of potential rain clouds by multispectral analysis of AVHRR data. Atmos. Res., 34, 259-283, https://doi.org/10.1016/0169-8095(94)90096-5.

Schmeissner, T., R. A. Shaw, J. Ditas, F. Stratmann, M. Wendisch, and H. Siebert, 2015: Turbulent mixing in shallow trade wind cumuli: Dependence on cloud life cycle. J. Atmos. Sci., 72, 1447-1465, https://doi.org/10.1175/JAS-D-14-0230.1.

Seifert, A., and T. Heus, 2013: Large-eddy simulation of organized precipitating trade wind cumulus clouds. Atmos. Chem. Phys., 13, 5631-5645, https://doi.org/10.5194/acp-13-5631-2013.

Siebesma, A. P., and Coauthors, 2003: A large eddy simulation intercomparison study of shallow cumulus convection. $J$. Atmos. Sci., 60, 1201-1219, https://doi.org/10.1175/15200469(2003)60<1201:ALESIS>2.0.CO;2.

Stevens, B., and Coauthors, 2005: Evaluation of large-eddy simulations via observations of nocturnal marine stratocumulus. Mon. Wea. Rev., 133, 1443-1462, https://doi.org/10.1175/ MWR2930.1.

Tas, E., A. Teller, O. Altaratz, D. Axisa, R. Bruintjes, Z. Levin, and I. Koren, 2015: The relative dispersion of cloud droplets: Its robustness with respect to key cloud properties. Atmos. Chem. Phys., 15, 2009-2017, https://doi.org/10.5194/acp-152009-2015.

Trenberth, K. E., J. T. Fasullo, and J. Kiehl, 2009: Earth's global energy budget. Bull. Amer. Meteor. Soc., 90, 311-324, https:// doi.org/10.1175/2008BAMS2634.1.

Wyszogrodzki, A. A., W. W. Grabowski, L. P. Wang, and O. Ayala, 2013: Turbulent collision-coalescence in maritime shallow convection. Atmos. Chem. Phys., 13, 8471-8487, https:// doi.org/10.5194/acp-13-8471-2013.

Yang, F., R. Shaw, and H. Xue, 2016: Conditions for super-adiabatic droplet growth after entrainment mixing. Atmos. Chem. Phys., 16, 9421-9433, https://doi.org/10.5194/acp-16-9421-2016.

Yeom, J. M., S. S. Yum, F. Mei, B. Schmid, J. Comstock, L. A. T. Machado, and M. A. Cecchini, 2019: Impact of secondary droplet activation on the contrasting cloud microphysical relationships during the wet and dry seasons in the Amazon. Atmos. Res., 230, 104648, https://doi.org/10.1016/j.atmosres. 2019.104648.

Zelinka, M. D., T. A. Myers, D. T. McCoy, S. Po-Chedley, P. M. Caldwell, P. Ceppi, S. A. Klein, and K. E. Taylor, 2020: Causes of higher climate sensitivity in CMIP6 models. Geophys. Res. Lett., 47, e2019GL085782, https://doi.org/10.1029/ 2019GL085782.

Zhang, S., H. Xue, and G. Feingold, 2011: Vertical profiles of droplet effective radius in shallow convective clouds. Atmos. Chem. Phys., 11, 4633-4644, https://doi.org/10.5194/acp-114633-2011.

Zhao, M., and P. H. Austin, 2005: Life cycle of numerically simulated shallow cumulus clouds. Part II: Mixing dynamics. $J$. Atmos. Sci., 62, 1291-1310, https://doi.org/10.1175/JAS3415.1. 